\title{
Compositional reflectance and transmittance model for multilayer specimens
}

\author{
Mathieu Hébert, ${ }^{1, *}$ Roger David Hersch, ${ }^{1}$ and Jean-Marie Becker ${ }^{2}$ \\ ${ }^{1}$ School for Computer and Communication Sciences, Ecole Polytechnique Fédérale de Lausanne (EPFL), \\ 1015 Lausanne, Switzerland \\ ${ }^{2}$ CPE-Lyon and Laboratoire TSI, UMR CNRS 5516, Saint-Etienne, France \\ *Corresponding author: mathieu.hebert@epfl.ch
}

Received January 12, 2007; revised April 18, 2007; accepted April 29, 2007; posted May 4, 2007 (Doc. ID 78952); published July 27, 2007

\begin{abstract}
We propose a compositional model for predicting the reflectance and the transmittance of multilayer specimens composed of layers having possibly distinct refractive indices. The model relies on the laws of geometrical optics and on a description of the multiple reflection-transmission of light between the different layers and interfaces. The highly complex multiple reflection-transmission process occurring between several superposed layers is described by Markov chains. An optical element such as a layer or an interface forms a biface. The multiple reflection-transmission process is developed for a superposition of two bifaces. We obtain general composition formulas for the reflectance and the transmittance of a pair of layers and/or interfaces. Thanks to these compositional expressions, we can calculate the reflectance and the transmittance of three or more superposed bifaces. The model is applicable to regular compositions of bifaces, i.e., multifaces having on each face an angular light distribution that remains constant along successive reflection and transmission events. Kubelka's layering model, Saunderson's correction of the Kubelka-Munk model, and the Williams-Clapper model of a color layer superposed on a diffusing substrate are special cases of the proposed compositional model. (C) 2007 Optical Society of America

OCIS codes: $000.3860,230.4170,120.5700,120.7000,330.1710$.
\end{abstract}

\section{INTRODUCTION}

Printed and painted colored specimens are generally composed of several layers having possibly distinct refractive indices. Light may be scattered and/or absorbed in the layers, as well as reflected and transmitted at the interfaces between layers of distinct refractive indices. The coloration of light interacting with a specimen results from the combined effects of scattering, absorption, reflection, and transmission, depending on the nature of the superposed layers, the angular distribution of light, and the geometry of observation. Existing reflectance and transmittance prediction models are dedicated to specific multilayer specimens. The reflectance and transmittance model we propose is applicable to all multilayer specimens comprising nonscattering and strongly scattering layers. Let us first present specific multilayer specimens and the corresponding classical spectral prediction models.

\section{A. Strongly Scattering Layers}

The well-known Kubelka-Munk theory [1,2] enables predicting the reflection and the transmission spectra of layers having at every point the same high-scattering and low-absorbing properties [3]. When several layers with identical refractive indices are superposed, their global reflectance and transmittance can be computed according to Kubelka's layering model [4] and expressed as functions of the individual layer reflectances and transmittances. Since the layers have the same refractive index and are strongly scattering, the light penetrating the multilayer loses its angular distribution and becomes im- mediately diffuse. Kubelka's multilayer reflectance and transmittance model is therefore independent of the angular distribution of the incident light.

Let us introduce Kubelka's layering model by considering two superposed strongly scattering layers having the same refractive index, identical to the refractive index of the surrounding medium. When light is incident on their upper side, the layers have a reflectance $R_{1}$ (resp. $R_{2}$ ) and a transmittance $T_{1}$ (resp. $T_{2}$ ). When light is incident on their lower side, they have a reflectance $R_{1}^{\prime}$ (resp. $R_{2}^{\prime}$ ) and a transmittance $T_{1}^{\prime}$ (resp. $T_{2}^{\prime}$ ). Figure 1 shows the multiple reflection-transmission process of light taking place within the bilayer, with the incident light coming at the upper side. By summing the different fractions of light emerging at the upper side, we obtain a geometric series expressing the bilayer's global reflectance $R$ :

$$
\begin{aligned}
R & =R_{1}+T_{1} R_{2} T_{1}^{\prime}+T_{1} R_{2} R_{1}^{\prime} R_{2} T_{1}^{\prime}+T_{1} R_{2}\left(R_{1}^{\prime} R_{2}\right)^{2} T_{1}^{\prime}+\cdots \\
& =R_{1}+T_{1} T_{1}^{\prime} R_{2} \frac{1}{1-R_{1}^{\prime} R_{2}} .
\end{aligned}
$$

The fractions of light emerging at the lower side also form a geometric series, expressing the bilayer's global transmittance $T$ :

$$
\begin{aligned}
T & =T_{1} T_{2}+T_{1} R_{2} R_{1}^{\prime} T_{2}+T_{1}\left(R_{2} R_{1}^{\prime}\right)^{2} T_{2}+\cdots \\
& =T_{1} T_{2} \frac{1}{1-R_{1}^{\prime} R_{2}}
\end{aligned}
$$




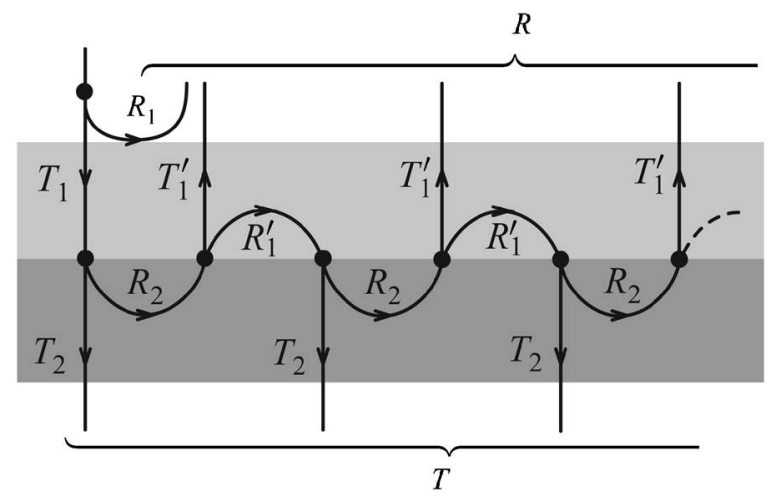

Fig. 1. Multiple reflection-transmission of light within two superposed nonsymmetrical layers.

\section{B. Saunderson Correction}

In most cases, the refractive index of the specimen is different from the one of air. Light is reflected and transmitted at both sides of the air-specimen interface, according to Snell's laws and Fresnel's formulas [5]. Saunderson [6] proposed a correcting formula giving the reflectance of a specimen interfaced with air as a function of the intrinsic reflectance of the specimen without interface. This correcting formula was established by describing the multiple reflections of light between the specimen and the interface. The same graph as in Fig. 1 may be used. Instead of two superposed layers, we have at the lower position a diffusing specimen of intrinsic reflectance $R$. At the upper position, we have the specimen-air interface, which has a reflectance $k_{1}$ and a transmittance $\left(1-k_{1}\right)$ at the air side, and a reflectance $k_{2}$ and a transmittance $\left(1-k_{2}\right)$ at the specimen side. The global reflectance $R^{\prime}$ of the specimen interfaced with air is expressed as in Eq. (1), i.e.,

$$
\begin{aligned}
R^{\prime} & =k_{1}+\left(1-k_{1}\right) R\left(1-k_{2}\right)+\left(1-k_{1}\right) R k_{2} R\left(1-k_{2}\right)+\cdots \\
& =k_{1}+\left(1-k_{1}\right)\left(1-k_{2}\right) \frac{R}{1-k_{2} R} .
\end{aligned}
$$

In the case of a sheet of a diffusing medium surrounded on both sides by a medium of distinct refractive index, we have a central diffusing layer bounded by two interfaces. The global reflectance and the transmittance of the slice can be obtained by describing the multiple reflectiontransmission process of light among these three elements [7-9].

\section{Diffusing Background Coated with a Transparent Layer}

Since it is restricted to strongly scattering layers, Kubelka's theory cannot be used for modeling a diffuse background coated with a nonscattering layer. A photograph, for example, is composed of a diffusing background (paper bulk) coated with a transparent possibly absorbing layer having the same refractive index as the diffusing background. One can follow the approach of Williams and Clapper and describe the multiple reflectiontransmission process between the diffuse background and the coating-air interface. The transparent coating may be grouped together with the coating-air interface so as to form a single light reflector-transmitter called colored interface. For directional incident light, the reflectance (resp. transmittance) of this colored interface is given by a directional reflection function (resp. transmission function) derived from Fresnel's formulas and Beer's law. When the incident light is diffuse, the reflectance (resp. transmittance) of the colored interface is obtained by summing up the contribution of each incident ray, i.e., by integrating angularly the directional reflection function (resp. transmission function) over the hemisphere $[9,10]$. For the colored interface superposed on a diffusing background, the reflectance and transmittance are computed in a similar manner as the Saunderson correction, i.e., by describing the multiple reflection-transmission process according to Fig. 1. The resulting analytical expression for the reflectance is similar to Eq. (3).

This approach was followed by Williams and Clapper [11], who considered directional incident light at $45^{\circ}$ and a radiance detector capturing light at $0^{\circ}\left(45^{\circ} / 0^{\circ}\right.$ measuring geometry). Shore and Spoonhower adapted the Williams-Clapper reflectance expression to other measuring geometries [12]. Simonot et al. [13] extended the Williams-Clapper model to the case where the coating is replaced by a stack of partly absorbing transparent layers having distinct refractive indices and flat interfaces. Even though light is multiply reflected and transmitted inside the transparent layer stack, according to Snell's laws, directional incident light remains directional without being diffused. The directional reflection and transmission functions are calculated as functions of the angle of incidence. For diffuse incident light, the diffuse reflectance and transmittance of the transparent layer stack are obtained by integrating angularly over the hemisphere the layer stack's directional reflection function (resp. the directional transmission function). The transparent layer stack may be superposed on a diffusing background. By describing the multiple reflection-transmission process of light between the transparent layer stack and the diffusing background, the analytical expression for the global reflectance of the specimen is derived in the same manner as when deriving Eq. (3).

\section{Prints}

The same multiple reflection-transmission approach can be applied to halftone prints. A halftone print is composed of a diffusing background (paper) and of a coating formed by juxtaposed colorant areas. We assume that the juxtaposed colorant areas form a single layer, called inked layer, which has the same refractive index as the paper background and has a flat interface with air. The transmission of light across the inked layer is attenuated by the colorants according to their surface ratio. A transparent inked layer is considered together with the ink-air interface and forms a colored interface as in the case of a photograph. The reflectance of the print is thus obtained by considering the multiple reflection-transmission process of light between this colored interface and the paper background. In a recent contribution [9], the reflectance and the transmittance of recto-verso halftone prints is modeled by considering the multiple reflectiontransmission of light among the central paper bulk layer, a colored interface at its upper side, and a different col- 
ored interface at its lower side. This model is also valid for paper sheets, whose colored interfaces are simply the paper-air interface.

The classical model introduced by Clapper and Yule for halftone prints [14] also relies on a multiple reflectiontransmission approach. However, light is assumed to have always the same angular distribution each time it crosses the inked layer. Thus, the attenuation of light due to absorption in the inked layer is modeled without consideration of its orientation $[9,10]$.

\section{E. Other Multilayer Specimens}

In all the models mentioned above, light is reflected and transmitted multiple times between less than three layers and/or interfaces. In the special case of specimens composed of several strongly scattering layers of identical refractive index, Kubelka's model enables applying iteratively the two-layer reflectance and transmittance formulas [4]. One computes the reflectance and the transmittance of two layers, then the reflectance and the transmittance of these two layers plus a third layer and so on.

However, in many specimens, we have an alternation of scattering and nonscattering layers possibly having different refractive indices. For example, when we fold a sheet of paper, we have a first sheet of paper, a central slice of air, and a second sheet of paper. Since paper and air have different refractive indices, each of the four paper-air interfaces creates light reflections and transmissions. For such a specimen, we should model the multiple reflection-transmission of light among these seven elements (two strongly scattering layers, one transparent layer, and four interfaces) by taking care of their different optical properties. Such a multiple reflectiontransmission process is extremely complex and very difficult to describe without an appropriate mathematical model.

\section{F. Compositional Approach}

We develop a methodology relying on Markov chains for modeling the multiple reflection-transmission process and for computing the reflectance and the transmittance of any multilayer specimen composed of scattering layers and/or nonscattering layers having possibly distinct refractive indices. In order to describe every multiple reflection-transmission process, we introduce a special formalism. We represent every layer and every interface between layers by the single concept of biface. Bifaces are characterized by their upper-side reflectance, their upperside transmittance, their lower-side reflectance, and their lower-side transmittance. These four terms are gathered into a $2 \times 2$ matrix called transfer matrix. Every multilayer specimen is seen as a superposition of bifaces and is called a multiface. For describing the multiple reflection-transmission process between the different bifaces, we use Markov chains. Their graphical representation as finite graphs provides a very synthetic overview of the multiple reflection-transmission process. Moreover, using the Markov matrix formalism, a single matrix computation is sufficient for obtaining the global reflectance and the global transmittance of multifaces for upper and lower illuminations. The computation method is independent of the number of superposed bifaces.

In practice, we need to compute the multiple reflectiontransmission process for only two bifaces. Thanks to the established matrix composition rules, we calculate the global transfer matrix of a multiface. The composition of two transfer matrices is associative. Thus, for multifaces composed of three or more layers, we can compose successively, two by two, the transfer matrices of the corresponding superposed bifaces and multifaces.

This paper is structured as follows. In Section 2, we recall the definitions of reflectance and transmittance. The concepts of biface and multiface are introduced in Section 3. We establish the correspondence between multiple reflection-transmission processes and Markov chains in Section 4. In Section 5, by applying Markov's theory, we develop the expressions for the reflectances and the transmittances of two superposed bifaces. The algebraic composition operation is defined in Section 6. In Section 7, we consider the case of three superposed bifaces and show the associativity of the composition operation. In Section 8 , we introduce a decomposition operation useful for separating the parameters depending on the illumination and measuring geometry. The application of the compositional model is illustrated in Section 9 by examples of prints on monolayer and multilayer supports. In Section 10, we draw the conclusions.

\section{REFLECTANCE AND TRANSMITTANCE}

Reflectance is defined as the ratio of a reflected flux to an incident flux, considering a surface element on the considered specimen, the angular distribution of the incident flux and the set of directions over which the reflected flux is observed [15]. The same definition also applies for transmittance in respect to the transmitted light flux.

A specimen receives directional incident light from direction $(\theta, \phi)$. It is observed over the whole hemisphere; i.e., all the reflected light is collected by the capturing device. We assume that the specimen is azimuthally isotropic; i.e., its reflection properties are independent of the azimuthal angle $\phi$. The specimen is thus characterized by its directional reflectance, $R(\theta)$, which is a function of the incident angle $\theta$.

Let us now determine the reflectance of the specimen illuminated with perfectly diffuse light, i.e., with a Lambertian irradiance $E_{i}$, and observed over the whole hemisphere. Since it is Lambertian [15], the incident irradiance is uniformly distributed over the hemisphere; i.e., the radiance incident from every direction $(\theta, \phi)$ of the hemisphere is $E_{i} / \pi$. Therefore, a surface element $\mathrm{d} s$ on the specimen receives from a given direction $(\theta, \phi)$, through the infinitesimal solid angle $\mathrm{d} \omega=\sin \theta \mathrm{d} \theta \mathrm{d} \phi$, the elemental flux $\mathrm{d}^{2} \Phi_{i}(\theta, \phi)$,

$$
\mathrm{d}^{2} \Phi_{i}(\theta, \phi)=\mathrm{d} s \frac{E_{i}}{\pi} \cos \theta \sin \theta \mathrm{d} \theta \mathrm{d} \phi .
$$

A fraction $R(\theta)$ of this incident elemental flux is reflected into the hemisphere, function $R$ being the directional reflectance of the specimen. The resulting reflected flux, 
divided by the surface element area $\mathrm{d} s$, yields an element of reflected irradiance $\mathrm{d} E_{r}(\theta, \phi)$ :

$$
\mathrm{d} E_{r}(\theta, \phi)=R(\theta) \frac{E_{i}}{\pi} \cos \theta \sin \theta \mathrm{d} \theta \mathrm{d} \phi
$$

In order to obtain the total irradiance reflected by the specimen, one accounts for all the directions of incidence of light, i.e., one sums up over the hemisphere the element of reflected irradiance $\mathrm{d} E_{r}(\theta, \phi)$ :

$$
E_{r}=\int_{\phi=0}^{2 \pi} \int_{\theta=0}^{\pi / 2} R(\theta) \frac{E_{i}}{\pi} \cos \theta \sin \theta \mathrm{d} \theta \mathrm{d} \phi .
$$

Since the specimen is azimuthally isotropic, the integrated terms do not depend on angle $\phi$. The integration according to $\phi$ yields a factor $2 \pi$. After rearranging, the ratio $E_{r} / E_{i}$ defines the Lambertian reflectance $r$ of the azimuthally isotropic specimen:

$$
r=\int_{\theta=0}^{\pi / 2} R(\theta) \sin 2 \theta \mathrm{d} \theta .
$$

Equation (7) specifies the diffuse reflectance of flat interfaces when $R(\theta)$ is the Fresnel reflectivity of the interface. It has been tabulated by Judd [16] for various refractive indices between 1 and 2. A similar definition can be formulated for the transmission of diffuse light.

\section{BIFACES AND MULTIFACES}

The layers and the interfaces forming a multilayer specimen are each responsible for the reflection and the transmission of light. However, except in the case of strongly scattering layers, they have angle-dependent reflectance and transmittance. In order to take into account these angular dependences in the multiple reflectiontransmission model, we introduce the biface concept and classify bifaces according to the angle-dependence of their reflectance and transmittance.

A biface represents a single layer or a single interface, whose reflection and transmission properties are azimuthally isotropic and may be modeled by geometrical optics (no interference, no diffraction, and no luminescence phenomena). Because bifaces may receive light on their two sides, they are the junction of two faces, each one being characterized by its reflectance and its transmittance for a given angular distribution of light.

We distinguish three categories of bifaces. Transparent bifaces are perfectly nonscattering. They reflect and transmit single-directional light components when the incident light is single directional. They correspond to perfectly nonscattering layers (transparent layers) and perfectly flat interfaces. Scattering bifaces scatter light as a function of the angular distribution of the incident light. Scattering layers and rough interfaces belong to this category. Lambertian bifaces are perfect diffusers that reflect and transmit Lambertian light components independently of the angular distribution of the incident light. Strongly scattering layers belong to this category. Let us characterize these three types of bifaces.

\section{A. Transparent Bifaces}

A transparent biface does not scatter light at all. It is characterized by its upper-face directional reflectance $R(\theta)$ and transmittance $T(\theta)$ and its lower-face directional reflectance $R^{\prime}(\theta)$ and transmittance $T^{\prime}(\theta)$. These four angular functions are gathered into a matrixlike notation called fundamental transfer matrix, with its elements placed as follows:

$$
\left[\begin{array}{cc}
T(\theta) & R(\theta) \\
R^{\prime}(\theta) & T^{\prime}(\theta)
\end{array}\right] .
$$

When the biface is illuminated according to a specific angular distribution on each of its two sides, its faces have specific reflectances and transmittances. We gather them into a particular transfer matrix, whose elements are at the same place as the ones of the fundamental transfer matrix. For example, let us consider the biface having the fundamental transfer matrix of Eq. (8). It receives on its upper face directional light at angle $\psi$, yielding as upper particular reflectance the directional reflectance $R(\psi)$ and as upper particular transmittance the directional transmittance $T(\psi)$. On the lower face, it receives Lambertian light. The lower particular reflectance is the Lambertian reflectance $r^{\prime}$, derived from the directional reflectance $R(\theta)$ according to Eq. (7):

$$
r^{\prime}=\int_{\theta=0}^{\pi / 2} R^{\prime}(\theta) \sin 2 \theta \mathrm{d} \theta .
$$

Likewise, the lower particular transmittance of the biface is the Lambertian transmittance $t^{\prime}$ defined as

$$
t^{\prime}=\int_{\theta=0}^{\pi / 2} T^{\prime}(\theta) \sin 2 \theta \mathrm{d} \theta
$$

Thus, the particular transfer matrix of the biface illuminated with directional light at angle $\psi$ on its upper face and with Lambertian light on its lower face is

$$
\left[\begin{array}{cc}
T(\psi) & R(\psi) \\
r^{\prime} & t^{\prime}
\end{array}\right]
$$

As an example of a transparent biface, we have the partly absorbing nonscattering layer called transparent layer. It is characterized by its normal transmittance $t$, which is the attenuation factor for directional light crossing it perpendicularly. Its reflectance is zero. According to Beer's law [17], since light crossing the layer at angle $\theta$ travels a path of relative length $1 / \cos \theta$, the directional transmittance $T(\theta)$ of the transparent layer for every angle $\theta$ is

$$
T(\theta)=t^{1 / \cos \theta} .
$$

Therefore, the transparent layer is characterized by the fundamental transfer matrix:

$$
\left[\begin{array}{cc}
t^{1 / \cos \theta} & 0 \\
0 & t^{1 / \cos \theta}
\end{array}\right] .
$$

A flat interface between two media having different refractive indices is also a transparent biface. Its directional reflectance and transmittance correspond to its Fresnel reflectivity and transmittivity, given by Fresnel's formu- 
las [5]. Labeling 0 and 1 the upper and lower surrounding media, respectively, we note $R_{01}(\theta)$ the interface reflectivity and $T_{01}(\theta)$ the interface transmittivity when light arrives at angle $\theta$ from medium 0 . We note $R_{10}(\theta)$ the reflectivity and $T_{10}(\theta)$ the transmittivity when light arrives at angle $\theta$ from medium 1 . The interface has the fundamental transfer matrix

$$
\left[\begin{array}{ll}
T_{01}(\theta) & R_{01}(\theta) \\
R_{10}(\theta) & T_{10}(\theta)
\end{array}\right]
$$

\section{B. Scattering Bifaces}

The category of scattering bifaces contains rough interfaces and weakly scattering bifaces. Similarly to the transparent bifaces, they are characterized by a fundamental transfer matrix, whose elements may be derived from the bidirectional reflectance distribution function (BRDF) and the bidirectional transmittance distribution function (BTDF) of their faces if these functions are known [10]. When the illuminations geometries are specified on their two faces, one can express their particular transfer matrix.

\section{Lambertian Bifaces}

Lambertian bifaces correspond to strongly scattering bifaces whose reflectance and transmittance do not depend on the angular distribution of the incident light. The reflected and the transmitted lights are perfectly diffuse, i.e., Lambertian. A Lambertian biface has an upper-side reflectance $\rho$ and a possibly different lower-side reflectance $\rho^{\prime}$. According to the nonpolarity of strongly scattering layers [4], it has single side-independent transmittance $\tau$. Its transfer matrix is invariant in respect to the upper-side and lower-side illumination geometries:

$$
\left[\begin{array}{cc}
\tau & \rho \\
\rho^{\prime} & \tau
\end{array}\right] .
$$

\section{Multifaces}

A multilayer specimen incorporating various superposed bifaces is called a multiface. Since it is able to reflect and transmit light like bifaces, it is also characterized by a transfer matrix called global transfer matrix. The global transfer matrix is defined for a given angular distribution of the incident light and a given observation solid angle at each side. We note its upper reflectance $R_{U}$, its upper transmittance $T_{U}$, its lower reflectance $R_{V}$, and its lower transmittance $T_{V}$. The global transfer matrix $\mathbf{G}$ of a multiface is defined as

$$
\mathbf{G}=\left[\begin{array}{ll}
T_{U} & R_{U} \\
R_{V} & T_{V}
\end{array}\right] .
$$

Transfer matrices are not matrices in the classical sense since classical matrix operations such as sum (+) or product $(\cdot)$ are not defined. In Section 6 , we create a special operation $\bigcirc$ called composition operation. When a biface having the transfer matrix $\mathbf{F}_{1}$ is located on top of a biface having the transfer matrix $\mathbf{F}_{2}$, the resulting multiface has the global transfer matrix noted $\mathbf{F}_{1} \bigcirc \mathbf{F}_{2}$.

\section{MULTIPLE REFLECTION-TRANSMISSION PROCESS AND MARKOV CHAINS}

A given multilayer specimen corresponds to a multiface. Its composing layers and interfaces are the different bifaces of the multiface. Each time a light flux strikes a face (i.e., a side of the biface), it is split into a reflected component and a transmitted component, each one being itself incident onto another face and being again split into reflected and transmitted components. The process according to which fluxes components are split within the multiface is called the multiple reflection-transmission process. When a face absorbs light, the sum of its reflectance and its transmittance is less than 1. Absorption is therefore taken into account.

Each face within the multiface receives flux components at various steps of the multiple reflectiontransmission process. Recall that, except for Lambertian faces, the reflectance and the transmittance of a given face generally depend on the angular distribution of the incident light. If the angular distribution of light evolves during the multiple reflection-transmission process, the face reflectance and transmittance may vary and become step dependent. The face is said to be irregular. A multiface containing at least one irregular face is an irregular multiface, and the whole multiple reflection-transmission process is qualified as step dependent. The multiple reflection-transmission approach developed here is not suitable for such a multiface, since it will neither yield geometrical series nor analytical expressions for its global reflectance and transmittance. We therefore limit our considerations to regular multifaces for which the multiple reflection-transmission process is step independent.

\section{A. Regular Multifaces}

Depending on their composition, we may distinguish three types of regular multifaces:

1. Multifaces comprising only Lambertian bifaces (i.e., strongly scattering layers). Every face has constant stepindependent reflectance and transmittance. This is the type of multiface considered in Kubelka's model [4].

2. An alternation of non-Lambertian bifaces, i.e., transparent or scattering bifaces, and of Lambertian bifaces. The two faces of the non-Lambertian bifaces receive constantly Lambertian fluxes from their neighboring Lambertian bifaces. The two faces of the Lambertian bifaces have invariant reflectance and transmittance independently of the angular distribution of light. Since the faces of every biface have a constant step-independent reflectance and transmittance, the multiface is regular. Several Lambertian bifaces may also be consecutive. Between two Lambertian bifaces, there must be at most one non-Lambertian biface, e.g., a transparent biface, or a weakly scattering biface. Consecutive non-Lambertian bifaces may be considered as one non-Lambertian biface, without however describing the multiple reflectiontransmission process of light taking place inside it.

3. Multifaces comprising only transparent bifaces, i.e., transparent layers and flat interfaces. Such multifaces are called transparent multifaces. They are regular only for directional incident light and therefore irregular when the incident light is diffuse. The regularity for directional 
incident light is due to the fact that, during the multiple reflection-transmission process, the successive components received by each layer and each interface are oriented according to step-independent angles determined according to Snell's laws (see Fig. 2). When the incident light is diffuse, its angular distribution is modified at each reflection or transmission due to the angular dependence of Fresnel's formulas and Beer's law. The successive light components received by a layer or by an interface have different, step-dependent, angular distributions. Therefore, for transparent bifaces illuminated by diffuse incident light, the multiple reflection-transmission process is time dependent and the transparent multiface is irregular.

\section{B. Markov Chains}

We now consider only regular multifaces and stepindependent multiple reflection-transmission processes. Regular multifaces are considered as a system in which flux units transit from face to face by reflection or transmission, are partially absorbed, and partially reach the detectors. A flux state corresponds to a face on which an incident flux unit is reflected or transmitted. A detector capturing an emerging flux unit corresponds also to a flux state. A flux unit moves from flux state to flux state at each reflection or transmission event, with the state transition probability corresponding to the considered reflectance (resp. transmittance). The path followed by a flux unit ends when it reaches an absorbing state, corresponding to a detector.

In a regular multiface, since each face has its specific step-independent reflectance and transmittance, each transition from one given state to another given state occurs with a same probability. We can therefore model the multiple reflection-transmission process as a homogenous discrete-time Markov chain [18]. Table 1 shows the analogy between the optical multiple reflectiontransmission processes and the corresponding terms of homogenous Markov chains.

The use of Markov chains provides two main advantages compared with the classical method of expressing multiple reflection-transmission processes by geometric series. First, the Markov matrix formalism allows the di-

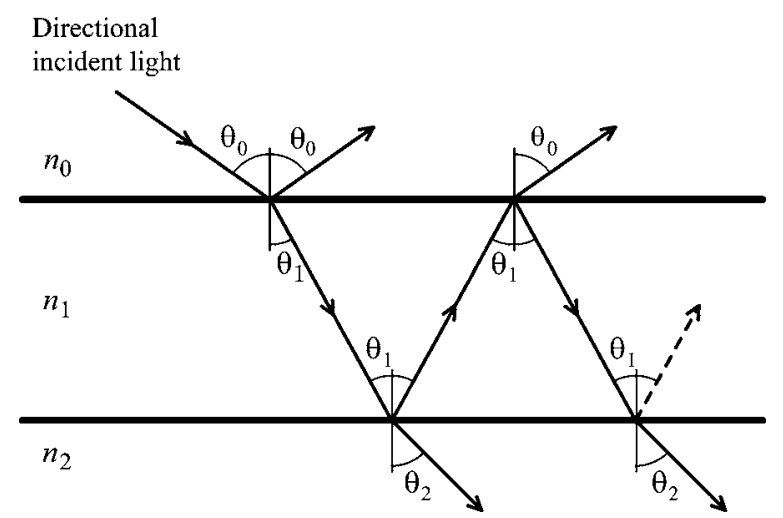

Fig. 2. Multiface representing a transparent layer bordered by two flat interfaces. When directional incident light illuminates such a multiface composed of transparent bifaces, every face receives directional light along step-independent orientations given by Snell's laws: $n_{0} \sin \theta_{0}=n_{1} \sin \theta_{1}=n_{2} \sin \theta_{2}$.
Table 1. Correspondence between Multiple Reflection-Transmission Processes and Markov Chains

\begin{tabular}{lc}
\hline \multicolumn{1}{c}{ Optics } & Markov Chains \\
\hline Multiface & Structured stochastic system \\
Portion of flux on a face & State \\
Portion of flux on a detector & Absorbing state \\
Reflection/transmission & Transition \\
Reflectance/transmittance & Transition probability \\
Step-independent process & Homogenous Markov chain \\
\hline
\end{tabular}

rect calculation of the global transfer matrix of multifaces. Their upper and lower reflectances and transmittances are obtained by a single matrix computation, which embeds the geometric series and therefore avoids the need to establish them explicitly. Second, the Markov graph provides a clear synthetic view of the multiple reflection-transmission process for any number of superposed bifaces, whereas classical light propagation graphs (e.g. Fig. 2 in Ref. [9]) are very difficult to draw for more than three superposed bifaces.

\section{REGULAR QUADRIFACE}

In order to illustrate the concepts of biface and transfer matrix and to show how Markov chains are used, we propose to consider a regular quadriface composed of two bifaces and therefore four faces. Such a quadriface may represent, for example, two strongly scattering layers having the same refractive index, also identical to the refractive index of the surrounding medium (no interfaces). A light source and a detector are placed both at the upper and the lower sides of the quadriface (Fig. 3). The global transfer matrix of the quadriface is the same as in Eq. (16), and the particular transfer matrices of the upper and lower bifaces are defined, respectively, as

$$
\left[\begin{array}{ll}
p_{u} & s_{u} \\
r_{u} & x_{u}
\end{array}\right], \quad\left[\begin{array}{ll}
x_{v} & r_{v} \\
s_{v} & p_{v}
\end{array}\right] .
$$

\section{A. Markov Chain of a Regular Quadriface}

The corresponding Markov chain is represented by the graph of Fig. 4. Flux states representing external faces,

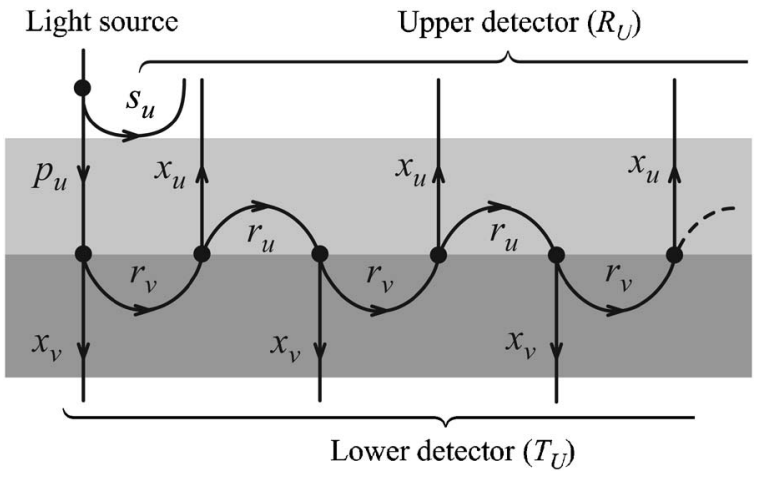

Fig. 3. Infinite graph showing the multiple reflectiontransmission of light within a quadriface, with light incident at the upper side (lower source off). 


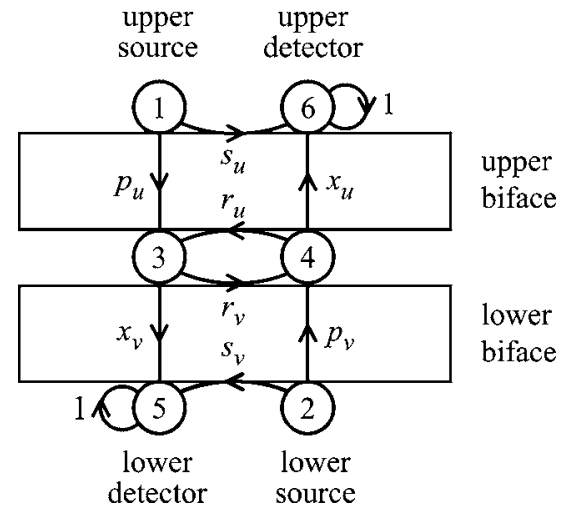

Fig. 4. Markov chain representing the multiple reflectiontransmission process within a quadriface.

which receive light from the light sources, are numbered 1 and 2. They are called ephemeral states since they are the starting point of a finite number of transitions. The detectors are represented by the absorbing states 5 and 6 . The loop connecting each detector with itself modelizes the fact that light captured by the detectors no longer interacts with the bifaces. Odd numbers (resp. even numbers) are attributed to the flux states receiving the light propagating downward (resp. upward). Odd numbers increase and even numbers decrease as the flux states are closer to the lower side. Note that flux-state labeling is arbitrary.

\section{B. Transition Probability Matrices}

Let $m_{i j}$ be the probability of transition from state $i$ to state $j$, which is zero if the two flux states are not directly connected. Matrix $\mathbf{M}=\left(m_{i j}\right)$ is called the single-step transition probability matrix of the Markov chain. For example, in this matrix, the transmittance $p_{u}$ of the upper biface represents the probability of transition from flux state 1 (line 1 of matrix) and flux state 3 (column 3 of matrix):

$$
\mathbf{M}=\left[\begin{array}{cccccc}
0 & 0 & p_{u} & 0 & 0 & s_{u} \\
0 & 0 & 0 & p_{v} & s_{v} & 0 \\
0 & 0 & 0 & r_{v} & x_{v} & 0 \\
0 & 0 & r_{u} & 0 & 0 & x_{u} \\
0 & 0 & 0 & 0 & 1 & 0 \\
0 & 0 & 0 & 0 & 0 & 1
\end{array}\right] .
$$

The evolution of the system may be observed step by step using a flux distribution vector, expressing the portions of light flux units being in the different flux states. In the following notations, we use bold characters for the two last elements of the vector, which represent the amounts of incident flux having reached the lower and the upper detectors, respectively. Let us first assume that the incident flux comes from the upper light source. At the origin, all the flux is in flux state 1; i.e., all the flux is incident on the upper face of the upper biface (see Fig. 4). The flux distribution vector is

$$
\mathbf{e}_{0}=\left[\begin{array}{llllll}
1, & 0,0,0, & \mathbf{0}
\end{array}\right] .
$$

At step 1, the flux distribution within the quadriface is given by the product $\mathbf{e}_{0} \cdot \mathbf{M}$ :

$$
\mathbf{e}_{1}=\mathbf{e}_{0} \cdot \mathbf{M}=\left[\begin{array}{llllll}
0, & 0, & p_{u}, & 0, & \mathbf{0}, \boldsymbol{s}_{\boldsymbol{u}}
\end{array}\right] .
$$

A fraction $s_{u}$ (external reflectance of the upper biface) of the incident light has already reached the upper detector (flux state 6 ). At step 2 , the product $\mathbf{e}_{1} \cdot \mathbf{M}$ shows that a fraction $p_{u} x_{v}$ has reached the lower detector (flux state 5):

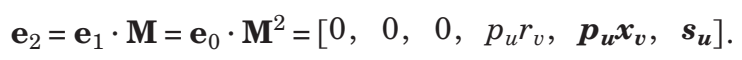

At step 3, the flux distribution vector is

$$
\begin{aligned}
\mathbf{e}_{3} & =\mathbf{e}_{2} \cdot \mathbf{M}=\mathbf{e}_{0} \cdot \mathbf{M}^{3} \\
& =\left[\begin{array}{llllll}
0, & 0, & p_{u} r_{u} r_{v}, & 0, & \boldsymbol{p}_{\boldsymbol{u}} \boldsymbol{x}_{\boldsymbol{v}}, & \boldsymbol{s}_{\boldsymbol{u}}+\boldsymbol{p}_{\boldsymbol{u}} \boldsymbol{r}_{\boldsymbol{v}} \boldsymbol{x}_{\boldsymbol{u}}
\end{array}\right] .
\end{aligned}
$$

Matrices $\mathbf{M}^{2}$ and $\mathbf{M}^{3}$ are called, respectively, the twostep and the three-step transition probability matrices. By continuing to perform the products of the flux distribution vector with matrix $\mathbf{M}$, one observes that the proportion of light reaching the detectors increases and that the proportion of light remaining in other states decreases. When the number of steps approaches infinity, no portion of the initial light flux remains within the quadriface. Therefore, the elements of the flux distribution vector $\mathbf{e}_{\infty}$ are zero, except the two last elements $m_{15}^{\infty}$ and $m_{16}^{\infty}$ :

$$
\mathbf{e}_{\infty}=\mathbf{e}_{0} \cdot \mathbf{M}_{\infty}=\left[\begin{array}{llllll}
0, & 0, & 0, & 0, & \boldsymbol{m}_{15}^{\infty}, & \boldsymbol{m}_{16}^{\infty}
\end{array}\right],
$$

with

$$
\mathbf{M}_{\infty}=\lim _{k \rightarrow \infty} \mathbf{M}^{k}
$$

These two elements correspond, respectively, to the fractions of the incident light flux being absorbed by flux states 5 and 6 , i.e., to the upper global transmittance and the upper global reflectance of the quadriface.

Let us now consider light coming from the lower source (state 2). At the origin, the flux distribution vector $\mathbf{e}_{0}$ is

$$
\mathbf{e}_{0}=\left[\begin{array}{llll}
0, & 0,0, \mathbf{0}, \mathbf{0}
\end{array}\right] \text {. }
$$

By multiplying this vector with the matrix $\mathbf{M}$ an infinite number of times, or by multiplying it by $\mathbf{M}_{\infty}$, we obtain a flux distribution vector whose elements are zeros, except the two last elements $m_{25}^{\infty}$ and $m_{26}^{\infty}$, which correspond, respectively, to the lower global reflectance and transmittance of the quadriface:

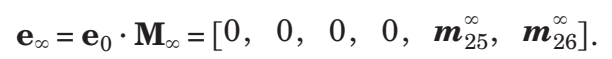

\section{Transfer Matrix of the Quadriface}

We conclude from Eqs. (20) and (23) that matrix $\mathbf{M}_{\infty}$ is of the form

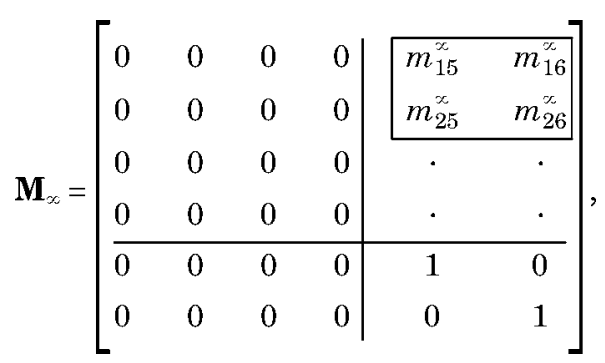

and that its upper-right $2 \times 2$ block corresponds to the global transfer matrix of the multiface: 


$$
\left[\begin{array}{cc}
T_{U} & R_{U} \\
R_{V} & T_{V}
\end{array}\right]=\left[\begin{array}{ll}
m_{15}^{\infty} & m_{16}^{\infty} \\
m_{25}^{\infty} & m_{26}^{\infty}
\end{array}\right] .
$$

Finally, according to the calculations performed in Appendix A, we obtain the following expression for the global transfer matrix of the quadriface:

$$
\left[\begin{array}{cc}
T_{U} & R_{U} \\
R_{V} & T_{V}
\end{array}\right]=\left[\begin{array}{cc}
\frac{p_{u} x_{v}}{1-r_{u} r_{v}} & s_{u}+\frac{p_{u} x_{u} r_{v}}{1-r_{u} r_{v}} \\
s_{v}+\frac{p_{v} x_{v} r_{u}}{1-r_{u} r_{v}} & \frac{p_{v} x_{u}}{1-r_{u} r_{v}}
\end{array}\right] .
$$

\section{COMPOSITION}

In order to show more clearly the relationship between the global transfer matrix of the quadriface given by Eq. (26) and the transfer matrices of the composing bifaces given in Eq. (17), we create an operation between transfer matrices, called a composition operation, noted $\bigcirc$, whose definition is

$$
\left[\begin{array}{cc}
p_{u} & s_{u} \\
r_{u} & x_{u}
\end{array}\right] \circ\left[\begin{array}{cc}
x_{v} & r_{v} \\
s_{v} & p_{v}
\end{array}\right]=\left[\begin{array}{cc}
\frac{p_{u} x_{v}}{1-r_{u} r_{v}} & s_{u}+\frac{p_{u} x_{u} r_{v}}{1-r_{u} r_{v}} \\
s_{v}+\frac{p_{v} x_{v} r_{u}}{1-r_{u} r_{v}} & \frac{p_{v} x_{u}}{1-r_{u} r_{v}}
\end{array}\right] .
$$

The left and right transfer matrices on the left part of the equation correspond, respectively, to the upper and the lower bifaces.

Equation (27) is called the quadriface formula. It is the most fundamental relation of the compositional model. It may be used each time two bifaces form a regular quadriface and embodies the multiple reflection-transmission process occurring between them.

Operation $\bigcirc$ is noncommutative, since a mutual exchange of position of two bifaces forms a different multiface having therefore different reflectances and transmittances. However, operation $\bigcirc$ is associative; i.e., for transfer matrices $\mathbf{F}_{1}, \mathbf{F}_{2}$, and $\mathbf{F}_{3}$, we have

$$
\left(\mathbf{F}_{1} \bigcirc \mathbf{F}_{2}\right) \bigcirc \mathbf{F}_{3}=\mathbf{F}_{1} \bigcirc\left(\mathbf{F}_{2} \bigcirc \mathbf{F}_{3}\right)=\mathbf{F}_{1} \bigcirc \mathbf{F}_{2} \bigcirc \mathbf{F}_{3} .
$$

This property appears clearly in the case of the hexaface presented in the next section.

\section{CASE OF A REGULAR HEXAFACE}

We consider a hexaface composed of three bifaces having the respective transfer matrices:

$$
\left[\begin{array}{cc}
p_{u} & s_{u} \\
r_{u} & x_{u}
\end{array}\right], \quad\left[\begin{array}{cc}
\tau & \rho \\
\rho^{\prime} & \tau^{\prime}
\end{array}\right], \quad\left[\begin{array}{ll}
x_{v} & r_{v} \\
s_{v} & p_{v}
\end{array}\right] .
$$

Such a hexaface may represent a sheet of glossy paper for which the three bifaces are the paper layer (Lambertian biface) and its two bordering flat interfaces with air (transparent bifaces). Since the Lambertian and nonLambertian bifaces are alternated, the hexaface is regular (category 2, Section 4). The central transfer matrix in Eq. (29) corresponds to the paper layer, and the two other transfer matrices correspond to its upper and its lower interfaces, respectively. The multiple reflectiontransmission process within the hexaface is represented by the Markov chain shown in Fig. 5 .

For calculating the global transfer matrix of the hexaface, we can develop a matrix computation similar to the one presented in the case of a quadriface (see Appendix B). But we can also use the composition operation and take benefit of its associativity. First, we select two consecutive bifaces and compose their transfer matrices using the quadriface formula (27):

$$
\left[\begin{array}{cc}
p_{u} & s_{u} \\
r_{u} & x_{u}
\end{array}\right] \bigcirc\left[\begin{array}{cc}
\tau & \rho \\
\rho^{\prime} & \tau^{\prime}
\end{array}\right]=\left[\begin{array}{cc}
\frac{p_{u} \tau}{1-r_{u} \rho} & s_{u}+\frac{p_{u} x_{u} \rho}{1-r_{u} \rho} \\
\rho^{\prime}+\frac{r_{u} \tau \tau^{\prime}}{1-r_{u} \rho} & \frac{x_{u} \tau^{\prime}}{1-r_{u} \rho}
\end{array}\right] .
$$

Then we compose the resulting transfer matrix with the one of the third biface by using again the quadriface formula (27). We obtain the hexaface formula:

$\left[\begin{array}{ll}p_{u} & s_{u} \\ r_{u} & x_{u}\end{array}\right] \bigcirc\left[\begin{array}{cc}\tau & \rho \\ \rho^{\prime} & \tau^{\prime}\end{array}\right] \bigcirc\left[\begin{array}{ll}x_{v} & r_{v} \\ s_{v} & p_{v}\end{array}\right]$

$$
=\left[\begin{array}{cc}
p_{u} x_{v} \frac{\tau}{D} & s_{u}+p_{u} x_{u} \frac{\rho-r_{v}\left(\rho \rho^{\prime}-\tau \tau^{\prime}\right)}{D} \\
s_{v}+p_{v} x_{v} \frac{\rho^{\prime}-r_{u}\left(\rho \rho^{\prime}-\tau \tau^{\prime}\right)}{D} & p_{v} x_{u} \frac{\tau^{\prime}}{D}
\end{array}\right],
$$

with $D=\left(1-r_{u} \rho\right)\left(1-r_{v} \rho^{\prime}\right)-r_{u} r_{v} \tau \tau^{\prime}$.

We can also compose the second and third transfer matrices:

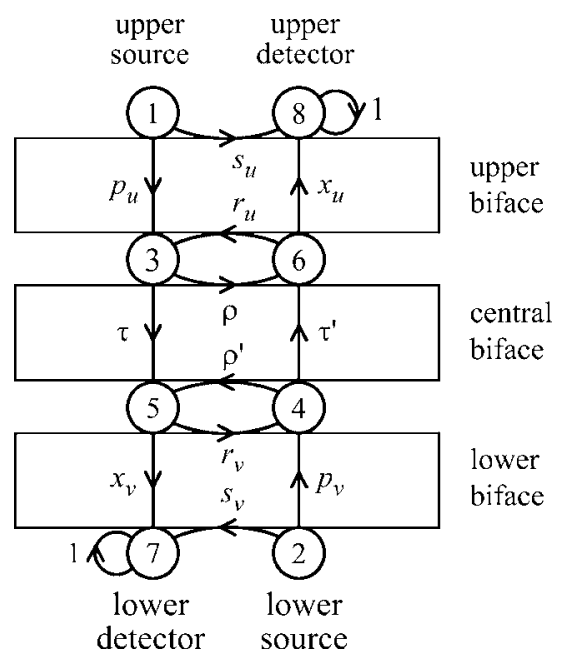

Fig. 5. Markov chain representing the multiple reflectiontransmission process in a hexaface. 


$$
\left[\begin{array}{cc}
\tau & \rho \\
\rho^{\prime} & \tau^{\prime}
\end{array}\right] \bigcirc\left[\begin{array}{cc}
x_{v} & r_{v} \\
s_{v} & p_{v}
\end{array}\right]=\left[\begin{array}{cc}
\frac{\tau x_{v}}{1-\rho r_{v}} & \rho+\frac{\tau \tau^{\prime} r_{v}}{1-\rho r_{v}} \\
s_{v}+\frac{p_{v} x_{v} \rho^{\prime}}{1-\rho r_{v}} & \frac{\tau^{\prime} p_{v}}{1-\rho r_{v}}
\end{array}\right]
$$

and then compose the resulting transfer matrix with the first transfer matrix. We obtain again the hexaface formula (31), which verifies the associativity of the composition operator $\bigcirc$. The matrix computed according to Markov's theory, presented in Appendix B, also yields the hexaface formula (31).

Thanks to the composition operation, it is no longer necessary to describe multiple reflection-transmission processes. Even for a regular multiface comprising many bifaces, we can compose them two by two in any order, provided that operator $\bigcirc$ is applied to the transfer matrices of consecutive bifaces.

\section{DECOMPOSITION OF A BIFACE}

Operator $\bigcirc$ may also be used for decomposing a biface into an abstract pair of bifaces whose composition is equal to the biface's transfer matrix. The biface is said to be decomposed. The upper (resp. lower) reflection arc is placed at the upper side of the upper biface (resp. the lower side of the lower biface). The other reflection arcs have a zero reflectance. The left (resp. right) transmission arc is placed at the left (resp. right) of the quadriface. We may choose to place it on the upper or the lower biface, with the other left arc (resp. right arc) having the transmittance 1 .

Figure 6 shows two examples of decompositions. For each example, we establish the transfer matrix of the two resulting bifaces and carry out the composition using the quadriface formula (27). In both cases, we retrieve the transfer matrix of the initial biface:

$\left[\begin{array}{ll}a & b \\ 0 & d\end{array}\right] \bigcirc\left[\begin{array}{ll}1 & 0 \\ c & 1\end{array}\right]=\left[\begin{array}{ll}1 & b \\ 0 & 1\end{array}\right] \bigcirc\left[\begin{array}{ll}a & 0 \\ c & d\end{array}\right]=\left[\begin{array}{ll}a & b \\ c & d\end{array}\right]$.

The two following decompositions are also possible:

$$
\left[\begin{array}{ll}
a & b \\
0 & 1
\end{array}\right] \bigcirc\left[\begin{array}{ll}
1 & 0 \\
c & d
\end{array}\right]=\left[\begin{array}{ll}
1 & b \\
0 & d
\end{array}\right] \bigcirc\left[\begin{array}{ll}
a & 0 \\
c & 1
\end{array}\right]=\left[\begin{array}{ll}
a & b \\
c & d
\end{array}\right] .
$$

Bifaces may be decomposed in order to separate the reflectance and transmittance terms that depend on the il- lumination and measuring geometry from the other terms. An example of decomposition is proposed in the next section for the case of prints.

\section{EXAMPLE OF APPLICATION}

We illustrate the application of the compositional model with the example of prints. Prints are multilayer specimens composed of a printing support, i.e., a sheet of diffusing substrate, and a coating incorporating the ink layer. The ink layer is assumed to have the same refractive index as the paper (no Fresnel reflections and transmissions at the substrate-ink interface), to be transparent and to have a flat interface with air.

According to our formalism, the print corresponds to four superposed bifaces: the flat air-ink interface (transparent biface) at the upper side, the ink layer (transparent biface), the layer of diffusing substrate (Lambertian biface), and the substrate-air interface (transparent biface) at the lower side. The flat air-ink interface and the ink layer are considered together as one transparent biface, called colored interface [9]. Thus, the colored interface (transparent biface), the substrate layer (Lambertian biface), and the substrate-air interface (transparent biface) form a regular hexaface belonging to category 2 . Their respective particular transfer matrices can be composed thanks to the composition operator $\bigcirc$.

A. Fundamental Transfer Matrix of the Colored Interface Although it is considered as being a single biface in the print, the colored interface is also a transparent multiface, which is regular for directional incident light (category 3, Section 4). The operator $\bigcirc$ can be applied for composing the fundamental transfer matrices of its two constituent transparent bifaces. Thus, in order to express the fundamental transfer matrix of the colored interface, let us compose the fundamental transfer matrix of the flat air-ink interface with the one of the ink layer.

The fundamental transfer matrices of the interface and the ink layer are, respectively, given by Eqs. (14) and (13). However, the orientations of light are different in the ink from in the air due to the refraction at the ink-air interface. According to Fig. 7, light is oriented at angle $\theta_{0}$ in air and at angle $\theta_{1}$ in the ink layer. They are related by Snell's law, i.e., $\sin \theta_{0}=n \sin \theta_{1}$, with $n$ being the refractive index of the ink. The corresponding fundamental transfer matrices of the interface and of the ink layer are, respectively,

$$
\left[\begin{array}{ll}
T_{01}\left(\theta_{0}\right) & R_{01}\left(\theta_{0}\right) \\
R_{10}\left(\theta_{1}\right) & T_{10}\left(\theta_{1}\right)
\end{array}\right],
$$

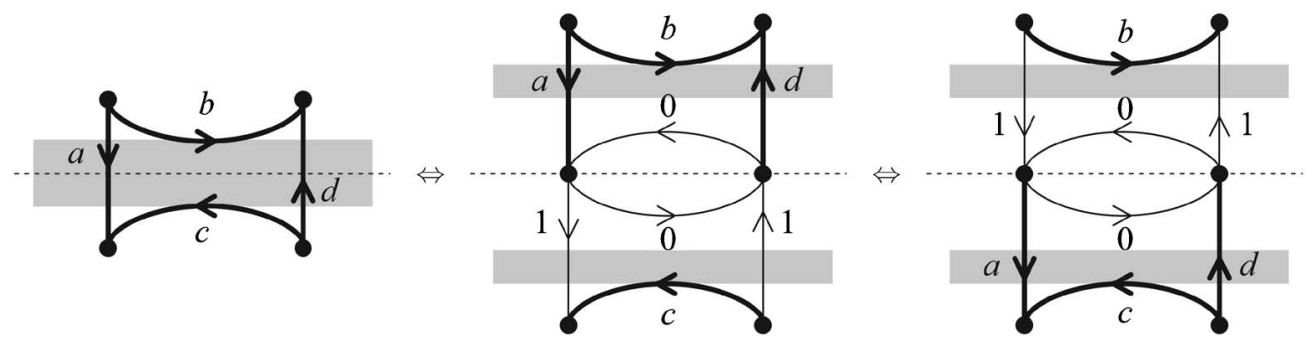

Fig. 6. Two possible decompositions of a biface. 


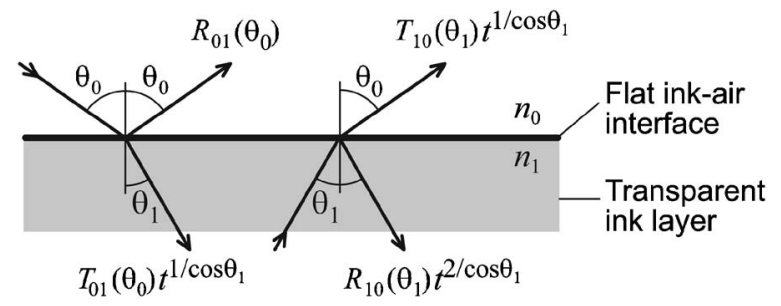

Fig. 7. Reflection and transmission of directional incident light at the upper side and the lower side of a colored interface, composed of an ink layer and its interface with air.

$$
\left[\begin{array}{cc}
t^{1 / \cos \theta_{1}} & 0 \\
0 & t^{1 / \cos \theta_{1}}
\end{array}\right]
$$

The colored interface is represented by the Markov chain of Fig. 8. The composition operation is applied to transfer matrices (35) and (36) and computed according to the following quadriface formula (27):

$$
\begin{gathered}
{\left[\begin{array}{cc}
T_{01}\left(\theta_{0}\right) & R_{01}\left(\theta_{0}\right) \\
R_{10}\left(\theta_{1}\right) & T_{10}\left(\theta_{1}\right)
\end{array}\right] \bigcirc\left[\begin{array}{cc}
t^{1 / \cos \theta_{1}} & 0 \\
0 & t^{1 / \cos \theta_{1}}
\end{array}\right]} \\
=\left[\begin{array}{cc}
T_{01}\left(\theta_{0}\right) t^{1 / \cos \theta_{1}} & R_{01}\left(\theta_{0}\right) \\
R_{10}\left(\theta_{1}\right) t^{2 / \cos \theta_{1}} & T_{10}\left(\theta_{1}\right) t^{1 / \cos \theta_{1}}
\end{array}\right] .
\end{gathered}
$$

The terms in the second row of matrix (37) are the lower transmittance and reflectance of the colored interface for directional light incident from below at angle $\theta_{1}$ (Fig. 7). The terms in the first row of matrix (37) are the upper transmittance and reflectance of the colored interface for directional light incident from air at angle $\theta_{0}$. We desire expressing them as functions of angle $\theta_{0}$ only. Since angles $\theta_{0}$ and $\theta_{1}$ are related by Snell's law, we have $\cos \theta_{1}=\sqrt{1-\left(\sin \theta_{0} / n\right)^{2}}$. Thus, the fundamental transfer matrix (37) of the colored interface becomes

$$
\left[\begin{array}{cc}
T_{01}\left(\theta_{0}\right) t^{1 / \sqrt{1-\left(\sin \theta_{0} / n\right)^{2}}} & R_{01}\left(\theta_{0}\right) \\
R_{10}\left(\theta_{1}\right) t^{2 / \cos \theta_{1}} & T_{10}\left(\theta_{1}\right) t^{1 / \cos \theta_{1}}
\end{array}\right] .
$$

\section{B. Composing the Bifaces of the Print}

The print is a regular hexaface composed of the colored interface at the upper position, the layer of diffusing substrate at the central position, and the substrate-air interface at the lower position. The corresponding Markov chain is shown in Fig. 9.

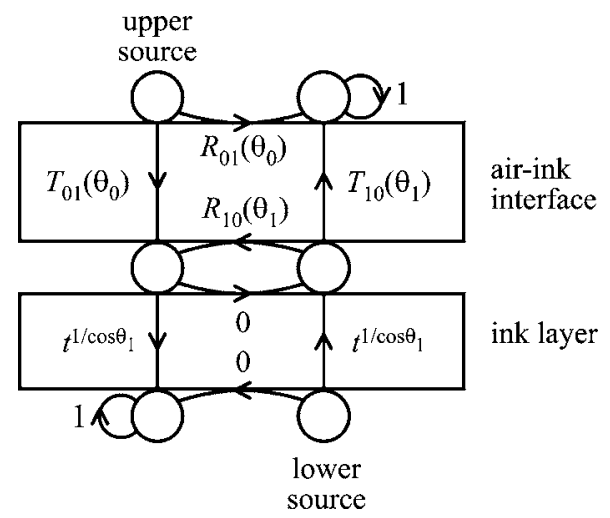

Fig. 8. Markov chain representing the multiple reflectiontransmission process of light in the colored interface.

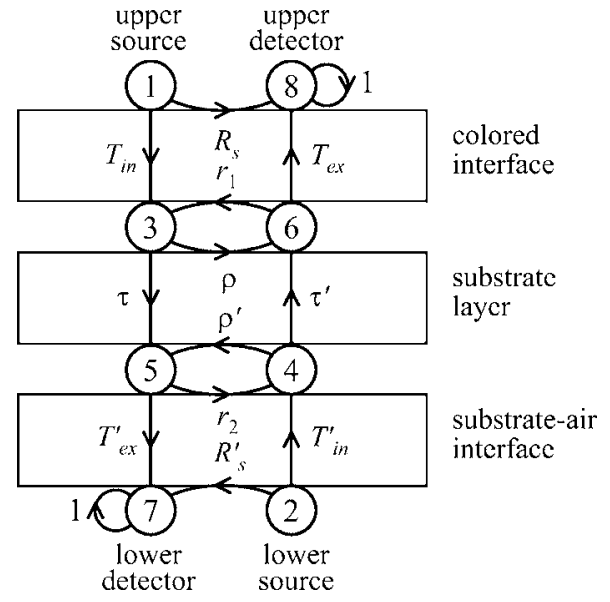

Fig. 9. Markov chain representing the multiple reflectiontransmission of light in a print.

The fundamental transfer matrix of the colored interface has been expressed in Eq. (38). According to the illumination and the measuring geometries used at the upper side, this fundamental transfer matrix is converted into a particular transfer matrix. Let us use the following generic notation for the particular transfer matrix of the upper-side colored interface:

$$
\left[\begin{array}{ll}
T_{i n} & R_{S} \\
r_{1} & T_{e x}
\end{array}\right]
$$

where $T_{\text {in }}$ and $R_{s}$ represent, respectively, the transmission and the reflection of the upper-side external incident light; $r_{1}$ represents the internal reflection of light at the substrate side; and $T_{e x}$ the transmission of light from below across the colored interface.

At the center of the print, the diffusing substrate (Lambertian biface) has an upper reflectance $\rho$, a lower reflectance $\rho^{\prime}$, and a transmittance $\tau$. It is characterized by the invariant transfer matrix:

$$
\left[\begin{array}{ll}
\tau & \rho \\
\rho^{\prime} & \tau
\end{array}\right]
$$

The flat substrate-air interface is characterized by its fundamental transfer matrix, whose elements are Fresnel reflectivities and transmittivities [see Eq. (14)]:

$$
\left[\begin{array}{ll}
T_{10}(\theta) & R_{10}(\theta) \\
R_{01}(\theta) & T_{01}(\theta)
\end{array}\right]
$$

where subscripts 0 and 1 denote the air and the substrate, respectively. As in the case of the colored interface located at the upper side, the particular transfer matrix of the lower-side substrate-air interface depends on the lower-side illuminating and measuring geometries. We use the following generic notation for the particular transfer matrix of the lower-side substrate-air interface:

$$
\left[\begin{array}{cc}
T_{e x}^{\prime} & r_{2} \\
R_{S}^{\prime} & T_{i n}^{\prime}
\end{array}\right]
$$

where $T_{e x}^{\prime}$ is the portion of the light transmitted by the interface and captured by the lower detector, $r_{2}$ is the substrate-side reflectance of the interface, $R_{s}$ is the re- 
flectance for the light incident at the air side, and $T_{\text {in }}$ is the attenuation of the external light coming from below.

The global transfer matrix of the recto-verso print is given by the following composition:

$$
\left[\begin{array}{cc}
T_{i n} & R_{s} \\
r_{1} & T_{e x}
\end{array}\right] \bigcirc\left[\begin{array}{cc}
\tau & \rho \\
\rho^{\prime} & \tau
\end{array}\right] \bigcirc\left[\begin{array}{cc}
T_{e x}^{\prime} & r_{2} \\
R_{s}^{\prime} & T_{i n}^{\prime}
\end{array}\right] .
$$

According to the hexaface formula (31), the composition gives the following expression for the global transfer matrix of the print:

$$
\left[\begin{array}{cc}
T_{i n} T_{e x}^{\prime} \frac{\tau}{D} & R_{s}+T_{i n} T_{e x} \frac{\rho-r_{2}\left(\rho \rho^{\prime}-\tau^{2}\right)}{D} \\
R_{s}^{\prime}+T_{i n}^{\prime} T_{e x}^{\prime} \frac{\rho^{\prime}-r_{1}\left(\rho \rho^{\prime}-\tau^{2}\right)}{D} & T_{i n}^{\prime} T_{e x} \frac{\tau^{\prime}}{D}
\end{array}\right]
$$

with $D=\left(1-r_{1} \rho\right)\left(1-r_{2} \rho^{\prime}\right)-r_{1} r_{2} \tau^{2}$.

We may verify that the expressions obtained for the upper global transmittance and reflectance of the rectoverso print [first line of matrix (44)] are similar to those obtained using the classical method of geometrical series [9], with $R_{s}=R_{s}^{\prime}=0$ as gloss is discarded from the measurements.

\section{Nonpolarity of the Internal Transmittance}

In the global transfer matrix of the print [Eq. (44)], the following terms depend on the illumination and measuring geometry: the external reflectances $R_{s}$ and $R_{s}^{\prime}$, the transmittances $T_{i n}$ and $T_{i n}^{\prime}$ relative to the penetrating light, and the transmittances $T_{e x}$ and $T_{e x}^{\prime}$ relative to the exit of light toward the detectors. In order to separate these terms from the rest of the multiple reflectiontransmission process, we perform a decomposition on the transfer matrices characterizing the upper colored interface and the lower substrate-air interface (see Section 8):

$$
\begin{aligned}
& {\left[\begin{array}{cc}
T_{i n} & R_{s} \\
r_{1} & T_{e x}
\end{array}\right]=\left[\begin{array}{cc}
T_{i n} & R_{s} \\
0 & T_{e x}
\end{array}\right] \bigcirc\left[\begin{array}{cc}
1 & 0 \\
r_{1} & 1
\end{array}\right],} \\
& {\left[\begin{array}{cc}
T_{e x}^{\prime} & r_{2} \\
R_{s}^{\prime} & T_{i n}^{\prime}
\end{array}\right]=\left[\begin{array}{cc}
1 & r_{2} \\
0 & 1
\end{array}\right] \bigcirc\left[\begin{array}{cc}
T_{e x}^{\prime} & 0 \\
R_{s}^{\prime} & T_{i n}^{\prime}
\end{array}\right] .}
\end{aligned}
$$

The global transfer matrix of the hexaface is thus given by the composition

$$
\begin{aligned}
& {\left[\begin{array}{cc}
T_{i n} & R_{s} \\
0 & T_{e x}
\end{array}\right] \bigcirc\left[\begin{array}{cc}
1 & 0 \\
r_{1} & 1
\end{array}\right] \bigcirc\left[\begin{array}{cc}
\tau & \rho \\
\rho^{\prime} & \tau
\end{array}\right] \bigcirc\left[\begin{array}{cc}
1 & r_{2} \\
0 & 1
\end{array}\right]} \\
& \bigcirc\left[\begin{array}{cc}
T_{e x}^{\prime} & 0 \\
R_{s}^{\prime} & T_{i n}^{\prime}
\end{array}\right]
\end{aligned}
$$

In Eq. (47), the three central transfer matrices contain only geometry-independent terms. By composing them using the hexaface formula (31), we obtain a new transfer matrix which is called the internal transfer matrix of the print

$$
\left[\begin{array}{cc}
\frac{\tau}{D} & \frac{\rho-r_{2}\left(\rho \rho^{\prime}-\tau^{2}\right)}{D} \\
\frac{\rho^{\prime}-r_{1}\left(\rho \rho^{\prime}-\tau^{2}\right)}{D} & \bar{D}
\end{array}\right]
$$

with $D=\left(1-r_{1} \rho\right)\left(1-r_{2} \rho^{\prime}\right)-r_{1} r_{2} \tau^{2}$.

The internal transfer matrix describes the geometryindependent part of the multiple reflection-transmission process. The two transmittance components, located on the diagonal of the internal transfer matrix, are equal. This property is called nonpolarity of internal transmittance. It extends to any regular multiface the principle of nonpolarity of the transmittance stated by Kubelka for intensely scattering layers [4]. The upper and lower global transmittances of the print differ only when different illumination and measuring geometries are used at the upper and the lower sides.

Obviously, by composing the internal transfer matrix with the most external transfer matrices of Eq. (47) and calculating the composition according to the hexaface formula Eq. (31), we retrieve the global transfer matrix (44) of the recto-verso print:

$$
\begin{aligned}
& {\left[\begin{array}{cc}
T_{i n} & R_{s} \\
0 & T_{e x}
\end{array}\right] \bigcirc\left[\begin{array}{cc}
\frac{\tau}{D} & \frac{\rho-r_{2}\left(\rho \rho^{\prime}-\tau^{2}\right)}{D} \\
\frac{\rho^{\prime}-r_{1}\left(\rho \rho^{\prime}-\tau^{2}\right)}{D} & \frac{\tau}{D}
\end{array}\right]} \\
& {\left[\begin{array}{cc}
T_{e x}^{\prime} & 0 \\
R_{s}^{\prime} & T_{i n}^{\prime}
\end{array}\right] .}
\end{aligned}
$$

\section{Example of Illumination and Measuring Geometries}

In Appendix C, we present the different possible expressions for the particular transfer matrices of a transparent biface according to the illumination geometry (directional incident light or Lambertian incident light) and the measuring geometry (radiance detector or integrating sphere). We propose here an example of geometry, where the upper light source is collimated light at $\psi=45^{\circ}$ and the upper capturing device is a radiance detector oriented at $\psi^{\prime}=0^{\circ}$. At the lower side, the light source is Lambertian and the capturing device is a radiance detector at $\psi^{\prime}=0^{\circ}$.

The colored interface is characterized by the fundamental transfer matrix given by Eq. (38). With the aid of Table 2 in Appendix C, we derive the following particular transfer matrix for the upper-side colored interface:

$$
\begin{aligned}
& {\left[\begin{array}{cc}
T_{\text {in }} & R_{S} \\
r_{1} & T_{\text {ex }}
\end{array}\right]} \\
& ={ }^{\operatorname{dir}\left(45^{\circ}\right) \downarrow}\left[\begin{array}{cc}
T_{01}(\theta) t^{1 / \sqrt{1-(\sin \theta / n)^{2}}} & R_{01}(\theta) \\
R_{10}(\theta) t^{2 / \cos \theta} & T_{10}(\theta) t^{1 / \cos \theta}
\end{array}\right]_{\uparrow L}^{\uparrow r a d\left(0^{\circ}\right)} \\
& =\left[\begin{array}{cc}
T_{01}\left(45^{\circ}\right) t^{n / \sqrt{n^{2}-1 / 2}} & 0 \\
r(t) & \frac{T_{10}(0) t}{\pi n^{2}}
\end{array}\right]
\end{aligned}
$$


where function $r(t)$ represents the internal reflectance of the colored interface $[11,19]$

$$
r(t)=\int_{\theta=0}^{\pi / 2} R_{10}(\theta) t^{2 / \cos \theta} \sin 2 \theta \mathrm{d} \theta .
$$

At the lower side, the substrate-air interface is characterized by the fundamental transfer matrix given by Eq. (41). According to Table 3 (Appendix C), its particular transfer matrix is

$$
\begin{aligned}
& {\left[\begin{array}{cc}
T_{e x}^{\prime} & r_{2} \\
R_{S}^{\prime} & T_{i n}^{\prime}
\end{array}\right]=\underset{r a d\left(\psi^{\prime}=0^{\circ}\right) \downarrow}{L \downarrow}\left[\begin{array}{ll}
T_{10}\left(\theta_{1}\right) & R_{10}\left(\theta_{1}\right) \\
R_{01}\left(\theta_{0}\right) & T_{01}\left(\theta_{0}\right)
\end{array}\right]_{\uparrow L}} \\
& =\left[\begin{array}{cc}
T_{10}\left(0^{\circ}\right) / \pi n^{2} & r_{10} \\
R_{01}\left(0^{\circ}\right) / \pi & t_{01}
\end{array}\right] \text {. }
\end{aligned}
$$

In Eq. (52), term $r_{10}$ represents the substrate-side diffuse

$$
\left[\begin{array}{c}
\frac{T_{01}(45) T_{10}(0)}{\pi n^{2}} t^{n / \sqrt{n^{2}-1 / 2}} \frac{\tau}{D} \\
\frac{R_{01}(0)}{\pi}+\frac{t_{01} T_{10}(0)}{\pi n^{2}} \cdot \frac{\rho^{\prime}-r_{10}(t)\left[\rho \rho^{\prime}-\tau^{2}\right]}{D}
\end{array}\right.
$$

with $D=\left[1-r_{10}(t) \rho\right] \cdot\left[1-r_{10} \rho^{\prime}\right]-r_{10}(t) r_{10} \tau^{2}$

\section{E. Multilayer Printing Support}

Let us now consider a different printing support composed of three superposed layers having the same refractive index. The central layer is nonscattering and the two other layers are strongly scattering. Such a printing support may be obtained by laminating opaque and transparent plastic sheets. Let us derive, using our compositional model, the transfer matrix of this new printing support, and then the global transfer matrix of the print.

The two strongly scattering layers correspond to Lambertian bifaces. Their invariant transfer matrices are, respectively,

$$
\left[\begin{array}{cc}
\tau_{1} & \rho_{1} \\
\rho_{1}^{\prime} & \tau_{1}
\end{array}\right], \quad\left[\begin{array}{ll}
\tau_{3} & \rho_{3} \\
\rho_{3}^{\prime} & \tau_{3}
\end{array}\right] .
$$

The central transparent layer has a normal transmittance $t_{2}$. Its fundamental transfer matrix is given by Eq. (13). It is at a central position within the multiface and receives Lambertian light on its two faces (see Appendix C). Its particular transfer matrix is given by Eq. (C.1):

$$
L \rightarrow\left[\begin{array}{cc}
t_{2}^{1 / \cos \theta} & 0 \\
0 & t_{2}^{1 / \cos \theta}
\end{array}\right]_{\leftarrow L}=\left[\begin{array}{cc}
\overline{t_{2}} & 0 \\
0 & \overline{t_{2}}
\end{array}\right],
$$

with reflectance of the substrate-air interface:

$$
r_{10}=\int_{\theta=0}^{\pi / 2} R_{10}(\theta) \sin 2 \theta \mathrm{d} \theta,
$$

and term $t_{01}$ represents its air-to-substrate diffuse transmittance [9]:

$$
t_{01}=\int_{\theta=0}^{\pi / 2} T_{01}(\theta) \sin 2 \theta \mathrm{d} \theta,
$$

The generic expression for the global transfer matrix of the print is given by Eq. (44). With the particular transfer matrices detailed in Eqs. (50) and (52), the global transfer matrix of the print becomes

$$
\left.\begin{array}{c}
\frac{T_{01}(45) T_{10}(0)}{\pi n^{2}} t^{1+n / \sqrt{n^{2}-1 / 2}} \cdot \frac{\rho-r_{10}\left[\rho \rho^{\prime}-\tau^{2}\right]}{D} \\
\frac{t_{01} T_{10}(0)}{\pi n^{2}} t \cdot \frac{\tau^{\prime}}{D}
\end{array}\right],
$$

The transfer matrix of the multilayer printing support is given by the following composition, calculated using the hexaface formula (31):

$$
\begin{aligned}
& {\left[\begin{array}{ll}
\tau_{1} & \rho_{1} \\
\rho_{1}^{\prime} & \tau_{1}
\end{array}\right] \bigcirc\left[\begin{array}{cc}
\overline{t_{2}} & 0 \\
0 & \overline{t_{2}}
\end{array}\right] \bigcirc\left[\begin{array}{cc}
\tau_{3} & \rho_{3} \\
\rho_{3}^{\prime} & \tau_{3}
\end{array}\right]} \\
& \quad=\left[\begin{array}{cc}
\tau_{1} \tau_{3} \frac{\overline{t_{2}}}{1-\rho_{1}^{\prime}{\overline{\rho_{3}}}^{2}} & \rho_{1}+\tau_{1}^{2} \frac{\rho_{3}{\overline{t_{2}}}^{2}}{1-\rho_{1}^{\prime} \bar{\rho}_{3} \bar{t}^{2}} \\
\rho_{3}^{\prime}+\tau_{3}^{2} \frac{\rho_{1}^{\prime}{\overline{t_{2}}}^{2}}{1-\rho_{1}^{\prime} \rho_{3}{\overline{t_{2}}}^{2}} & \tau_{1} \tau_{3} \frac{\overline{t_{2}}}{1-\rho_{1}^{\prime} \rho_{3} \overline{t 2}^{2}}
\end{array}\right] .
\end{aligned}
$$

When an ink layer is deposited on top of such a printing support, we obtain a multiface composed of the following bifaces: the colored interface, which comprises the air-ink interface and the ink layer; the first diffusing layer; the transparent layer; the second diffusing layer; and the backing layer-air interface. In this multiface, transparent and Lambertian bifaces are alternated. Therefore, it is regular (see Section 4). Its global transfer matrix results from the following composition:

$$
\begin{gathered}
{\left[\begin{array}{cc}
T_{\text {in }} & R_{s} \\
r_{1} & T_{e x}
\end{array}\right] \bigcirc\left[\begin{array}{cc}
\tau_{1} & \rho_{1} \\
\rho_{1}^{\prime} & \tau_{1}
\end{array}\right] \bigcirc\left[\begin{array}{cc}
\overline{t_{2}} & 0 \\
0 & \overline{t_{2}}
\end{array}\right]} \\
\bigcirc\left[\begin{array}{cc}
\tau_{3} & \rho_{3} \\
\rho_{3}^{\prime} & \tau_{3}
\end{array}\right] \bigcirc\left[\begin{array}{cc}
T_{e x}^{\prime} & r_{2} \\
R_{s}^{\prime} & T_{\text {in }}^{\prime}
\end{array}\right] .
\end{gathered}
$$

This composition may be calculated by applying four 
times the quadriface formula, or twice the hexaface formula.

With the same simplicity, we may consider that the central layer within the printing support is weakly scattering instead of being transparent. It is also illuminated on its two sides by Lambertian light. Its particular transfer matrix is of the form

$$
\left[\begin{array}{cc}
\tau_{2} & \rho_{2} \\
\rho_{2}^{\prime} & \tau_{2}
\end{array}\right]
$$

The global transfer matrix of the print is then given by the compositional equation (58), with transfer matrix (59) in place of transfer matrix (57). Note that reflectances $\rho_{2}$ and $\rho_{2}^{\prime}$ and transmittance $\tau_{2}$ may be deduced from the $\mathrm{BRDF}$ and the BTDF of the scattering layer if these functions are known [10]. They may also be indirectly deduced from global reflectance and transmittance measurements of the multilayer printing support if the parameters of the other layers are known.

\section{CONCLUSIONS}

The compositional model is a generalization of existing light reflection-transmission models. It allows predicting the reflectance and transmittance of specimens composed of several layers having different refractive indices. In order to unify the notions of layer and interface between layers of different refractive indices, we introduce the concept of biface. A biface reflects and transmits light on its top and bottom sides. We associate to every biface a transfer matrix that expresses the upper and the lower reflectances and transmittances according to the laws of radiometry and geometrical optics (Snell's laws, Fresnel's formulas, Beer's law). Multifaces are formed by superposing bifaces.

A multiface is regular if on each of its faces, the angular light distribution is constant along successive reflections and transmissions. This condition is fulfilled with any superposition of transparent and/or strongly diffusing bifaces. Multifaces comprising weakly scattering layers and/or rough interfaces are only regular if they are bordered by strongly diffusing bifaces.

Homogeneous discrete-time Markov chains are particularly adapted for modeling the multiple reflectiontransmission process of light within regular multilayer specimens. Light flux elements are repeatedly transmitted and reflected at the faces of a multiface and eventually reach the detectors. The reflectances and transmittances of a quadriface are obtained by establishing the Markov transition probability matrix whose transition probabilities correspond to the reflectances and transmittances of the bifaces forming the quadriface. The stationary distribution of the Markov chain yields the upper and lower global reflectances and transmittances of the quadriface. The resulting biface composition operation is associative and can therefore be repeatedly applied in order to obtain the transfer matrix of a multiface.

The computation of global reflectances and transmittances is first carried out with generic expressions that can then be particularized for specific light illumination and capturing conditions. The biface composition opera- tion also enables decomposing a multiface into an illumination and capturing geometry-independent part expressing internal reflectances and transmittances. The geometry-independent internal transmittances are the same for the upper and lower side. This generalizes to regular multifaces the principle of nonpolarity of transmittance stated by Kubelka [4] for strongly scattering layers.

Kubelka's model [4], the Williams-Clapper model [11], the Saunderson correction of the Kubelka-Munk model [6], the model of Simonot et al. [13], the model of Hébert and Hersch for recto-verso halftone prints [9] have each been developed for predicting the reflectance and/or the transmittance of a certain type of specimen. Their respective expressions can be retrieved with the present compositional model [10]. We show the simplicity of use of the compositional model by modeling prints on monolayer or multilayer printing supports. The proposed compositional model is also applicable to many other types of multilayer specimens, e.g., successions of nonscattering or/and strongly scattering layers having different refractive indices.

\section{APPENDIX A: DETAILED CALCULATION OF THE QUADRIFACE FORMULA}

In Section 6, we present a regular quadriface composed of two bifaces having the respective transfer matrices

$$
\left[\begin{array}{ll}
p_{u} & s_{u} \\
r_{u} & x_{u}
\end{array}\right], \quad\left[\begin{array}{ll}
x_{v} & r_{v} \\
s_{v} & p_{v}
\end{array}\right] .
$$

Let us derive the global transfer matrix of this regular quadriface. The multiple reflection-transmission process is described by the Markov chain represented in Fig. 4. The following single-step transition probability matrix is attached to this Markov chain:

$$
\mathbf{M}=\left(\begin{array}{cccc:cc}
0 & 0 & p_{u} & 0 & 0 & s_{u} \\
0 & 0 & 0 & p_{v} & s_{v} & 0 \\
0 & 0 & 0 & r_{v} & x_{v} & 0 \\
0 & 0 & r_{u} & 0 & 0 & x_{u} \\
\hdashline 0 & 0 & 0 & 0 & 1 & 0 \\
0 & 0 & 0 & 0 & 0 & 1
\end{array}\right) .
$$

Let us decompose $\mathbf{M}$ into submatrices $\mathbf{A}$ and $\mathbf{B}$ :

$$
\mathbf{M}=\left(\begin{array}{c|c}
\mathbf{A} & \mathbf{B} \\
\hline \mathbf{0}_{24} & \mathbf{I}
\end{array}\right)
$$

The two-step transition probability matrix is

$$
\mathbf{M}^{2}=\left(\begin{array}{cc}
\mathbf{A} & \mathbf{B} \\
\mathbf{0} & \mathbf{I}
\end{array}\right) \cdot\left(\begin{array}{cc}
\mathbf{A} & \mathbf{B} \\
\mathbf{0} & \mathbf{I}
\end{array}\right)=\left(\begin{array}{cc}
\mathbf{A}^{2} & \mathbf{A B}+\mathbf{B} \\
\mathbf{0} & \mathbf{I}
\end{array}\right) .
$$

The three-step transition probability matrix is

$$
\mathbf{M}^{3}=\left(\begin{array}{cc}
\mathbf{A}^{2} & \mathbf{A B}+\mathbf{B} \\
\mathbf{0} & \mathbf{I}
\end{array}\right) \cdot\left(\begin{array}{cc}
\mathbf{A} & \mathbf{B} \\
\mathbf{0} & \mathbf{I}
\end{array}\right)=\left(\begin{array}{cc}
\mathbf{A}^{3} & \mathbf{A}^{2} \mathbf{B}+\mathbf{A B}+\mathbf{B} \\
\mathbf{0} & \mathbf{I}
\end{array}\right) .
$$

Pursuing iteratively the products of $\mathbf{M}^{k}$ by $\mathbf{M}$, we obtain the following $k$-step transition probability matrix: 


$$
\mathbf{M}^{k}=\left(\begin{array}{cc}
\mathbf{A}^{k} & \sum_{j=0}^{k-1} \mathbf{A}^{j} \mathbf{B} \\
\mathbf{0} & \mathbf{I}
\end{array}\right),
$$

and thereby, according to its defining Eq. (21), the following infinite-step transition probability matrix:

$$
\mathbf{M}_{\infty}=\left(\begin{array}{cc}
\lim _{k \rightarrow \infty} \mathbf{A}^{k} & \sum_{j=0}^{\infty} \mathbf{A}^{j} \mathbf{B} \\
\mathbf{0} & \mathbf{I}
\end{array}\right) .
$$

In Eq. (A.1), either $r_{u}$ or $r_{v}$ is strictly less than 1. Otherwise, with $r_{u}=r_{v}=1$, we would have $x_{u}=x_{v}=0$ since the sum of the transmitted and reflected fractions of fluxes cannot exceed 1 . This would mean that light cannot exit the multiface. Hence, block $\mathbf{A}$ is a substochastic matrix [18]. The power of matrix $\mathbf{A}$ converges toward the zero matrix [10], and the upper-right matrix geometric series converges toward $\left(\mathbf{I}_{4}-\mathbf{A}\right)^{-1} \mathbf{B}$, where $\mathbf{I}_{4}$ is the identity matrix having the size of A. Thus, Eq. (A.3) becomes [20]

$$
\mathbf{M}_{\infty}=\left(\begin{array}{cc}
\mathbf{0} & \left(\mathbf{I}_{4}-\mathbf{A}\right)^{-1} \mathbf{B} \\
\mathbf{0} & \mathbf{I}
\end{array}\right) .
$$

The global transfer matrix $\mathbf{G}$ is the upper-right $2 \times 2$ block of $\mathbf{M}_{\infty}$ and therefore the upper $2 \times 2$ block of $\left(\mathbf{I}_{4}-\mathbf{A}\right)^{-1} \mathbf{B}$ :

$$
\left(\begin{array}{c}
\mathbf{G} \\
.
\end{array}\right)=\left(\mathbf{I}_{4}-\mathbf{A}\right)^{-1} \mathbf{B}
$$

where the dot represents a $2 \times 2$ block.

Let us now decompose blocks $\mathbf{A}$ and $\mathbf{B}$ into $2 \times 2$ blocks:

$$
\mathbf{M}=\left(\begin{array}{c|c}
\mathbf{A} & \mathbf{B} \\
\hline \mathbf{0}_{24} & \mathbf{I}
\end{array}\right)=\left(\begin{array}{c:c|c}
\mathbf{0} & \mathbf{P} & \mathbf{S} \\
\hdashline \mathbf{0} & \mathbf{R} & \mathbf{X} \\
\hline \mathbf{0} & \mathbf{0} & \mathbf{I}
\end{array}\right)=\left(\begin{array}{cc:cc|cc}
0 & 0 & p_{u} & 0 & 0 & s_{u} \\
0 & 0 & 0 & p_{v} & s_{v} & 0 \\
\hdashline 0 & 0 & 0 & r_{v} & x_{v} & 0 \\
0 & 0 & r_{u} & 0 & 0 & x_{u} \\
\hline 0 & 0 & 0 & 0 & 1 & 0 \\
0 & 0 & 0 & 0 & 0 & 1
\end{array}\right) .
$$

We have

$$
\left(\mathbf{I}_{4}-\mathbf{A}\right)=\left(\begin{array}{cc}
\mathbf{I} & -\mathbf{P} \\
\mathbf{0} & \mathbf{I}-\mathbf{R}
\end{array}\right) .
$$

Using a straightforward block computation, we obtain

$$
\left(\begin{array}{cc}
\mathbf{I} & -\mathbf{P} \\
\mathbf{0} & \mathbf{I}-\mathbf{R}
\end{array}\right) \cdot\left(\begin{array}{cc}
\mathbf{I} & \mathbf{P}(\mathbf{I}-\mathbf{R})^{-1} \\
\mathbf{0} & (\mathbf{I}-\mathbf{R})^{-1}
\end{array}\right)=\left(\begin{array}{cc}
\mathbf{I} & \mathbf{0} \\
\mathbf{0} & \mathbf{I}
\end{array}\right) .
$$

Therefore,

$$
\left(\mathbf{I}_{4}-\mathbf{A}\right)^{-1}=\left(\begin{array}{cc}
\mathbf{I} & \mathbf{P}(\mathbf{I}-\mathbf{R})^{-1} \\
\mathbf{0} & (\mathbf{I}-\mathbf{R})^{-1}
\end{array}\right) .
$$

Using the decomposition of $\mathbf{B}$ as a function of blocks $\mathbf{S}$ and $\mathbf{X}$, we have

$$
\left(\mathbf{I}_{4}-\mathbf{A}\right)^{-1} \mathbf{B}=\left(\begin{array}{cc}
\mathbf{I} & \mathbf{P}(\mathbf{I}-\mathbf{R})^{-1} \\
\mathbf{0} & (\mathbf{I}-\mathbf{R})^{-1}
\end{array}\right) \cdot\left(\begin{array}{l}
\mathbf{S} \\
\mathbf{X}
\end{array}\right)=\left(\begin{array}{c}
\mathbf{S}+\mathbf{P}(\mathbf{I}-\mathbf{R})^{-1} \mathbf{X} \\
(\mathbf{I}-\mathbf{R})^{-1} \mathbf{X}
\end{array}\right) .
$$

$\left(\mathbf{I}_{4}-\mathbf{A}\right)^{-1} \mathbf{B}$ is a $4 \times 2$ matrix whose upper $2 \times 2$ block corresponds to the global transfer matrix $\mathbf{G}$, i.e.,

$$
\mathbf{G}=\mathbf{S}+\mathbf{P}(\mathbf{I}-\mathbf{R})^{-1} \mathbf{X} .
$$

According to the decomposition defined in Eq. (A.4), matrix $\mathbf{I}-\mathbf{R}$ is expanded as

$$
\mathbf{I}-\mathbf{R}=\left(\begin{array}{ll}
1 & 0 \\
0 & 1
\end{array}\right)-\left(\begin{array}{cc}
0 & r_{v} \\
r_{u} & 0
\end{array}\right)=\left(\begin{array}{cc}
1 & -r_{v} \\
-r_{u} & 1
\end{array}\right) .
$$

Its determinant is $\Delta=1-r_{u} r_{v}>0$ since $r_{u} r_{v}<1$, and its inverse is

$$
(\mathbf{I}-\mathbf{R})^{-1}=\frac{1}{\Delta}\left(\begin{array}{cc}
1 & r_{v} \\
r_{u} & 1
\end{array}\right) .
$$

By inserting Eq. (A.6) into Eq. (A.5), one finally obtains the quadriface formula:

$$
\begin{aligned}
\mathbf{G} & =\left(\begin{array}{cc}
0 & s_{u} \\
s_{v} & 0
\end{array}\right)+\frac{1}{\Delta}\left(\begin{array}{cc}
p_{u} & 0 \\
0 & p_{v}
\end{array}\right) \cdot\left(\begin{array}{cc}
1 & r_{v} \\
r_{u} & 1
\end{array}\right) \cdot\left(\begin{array}{cc}
x_{v} & 0 \\
0 & x_{u}
\end{array}\right) \\
& =\left(\begin{array}{cc}
\frac{p_{u} x_{v}}{1-r_{u} r_{v}} & s_{u}+\frac{p_{u} x_{u} r_{v}}{1-r_{u} r_{v}} \\
s_{v}+\frac{p_{v} x_{v} r_{u}}{1-r_{u} r_{v}} & \frac{p_{v} x_{u}}{1-r_{u} r_{v}}
\end{array}\right) .
\end{aligned}
$$

\section{APPENDIX B: DETAILED CALCULATION OF THE HEXAFACE FORMULA}

In Section 7, we present a hexaface whose bifaces have the transfer matrices given by Eq. (29). The Markov chain is represented by the graph of Fig. 5. The single-step transition probability matrix $\mathbf{M}$ of the chain is obtained by placing at line $i$ and column $j$ the probability of transition from state $i$ to state $j$ :

$$
\mathbf{M}=\left(\begin{array}{cccccc:cc}
0 & 0 & p_{u} & 0 & 0 & 0 & 0 & s_{u} \\
0 & 0 & 0 & p_{v} & 0 & 0 & s_{v} & 0 \\
0 & 0 & 0 & 0 & \tau & \rho & 0 & 0 \\
0 & 0 & 0 & 0 & \rho^{\prime} & \tau^{\prime} & 0 & 0 \\
0 & 0 & 0 & r_{v} & 0 & 0 & x_{v} & 0 \\
0 & 0 & r_{u} & 0 & 0 & 0 & 0 & x_{u} \\
\hdashline 0 & 0 & 0 & 0 & 0 & 0 & 1 & 0 \\
0 & 0 & 0 & 0 & 0 & 0 & 0 & 1
\end{array}\right) .
$$

In the same way as in the quadriface, we can observe the step-by-step evolution of the system by using a flux distribution vector. Again, the amount of light that transits after an infinite number of steps from a light source to a detector is an element of the infinite-step transition probability matrix $\mathbf{M}_{\infty}$, defined by Eq. (21). In order to compute matrix $\mathbf{M}_{\infty}$, we decompose matrix $\mathbf{M}$ in the same manner as in Eq. (A.2), with blocks $\mathbf{A}$ and $\mathbf{B}$ subsequently subdivided into $2 \times 2$ blocks: 


$$
\begin{aligned}
& \mathbf{A}=\left(\begin{array}{cc|c}
\mathbf{0} & \mathbf{P} & \mathbf{0} \\
\hdashline & \mathbf{0} & \mathbf{C} \\
\hdashline \mathbf{0} & \mathbf{R} & \mathbf{0}
\end{array}\right)=\left(\begin{array}{cc:cc|cc}
0 & 0 & p_{u} & 0 & 0 & 0 \\
0 & 0 & 0 & p_{v} & 0 & 0 \\
\hdashline 0 & 0 & 0 & 0 & \tau & \rho \\
0 & 0 & 0 & 0 & \rho^{\prime} & \tau^{\prime} \\
\hline 0 & 0 & 0 & r_{v} & 0 & 0 \\
0 & 0 & r_{u} & 0 & 0 & 0
\end{array}\right), \\
& \mathbf{B}=\left(\begin{array}{c}
\mathbf{S} \\
\overline{\mathbf{0}} \\
\overline{\mathbf{X}}
\end{array}\right)=\left(\begin{array}{cc}
0 & s_{u} \\
s_{v} & 0 \\
-0 & 0 \\
0 & 0 \\
\hdashline x_{v} & 0 \\
0 & x_{u}
\end{array}\right)
\end{aligned}
$$

Either $r_{u}$ or $r_{v}$ is strictly less than 1 and block $\mathbf{A}$ is a substochastic matrix. Following the same reasoning line as for the quadriface, we find

$$
\mathbf{M}_{\infty}=\left(\begin{array}{cc}
\mathbf{0} & \left(\mathbf{I}_{6}-\mathbf{A}\right)^{-1} \mathbf{B} \\
\mathbf{0} & \mathbf{I}
\end{array}\right) .
$$

The global transfer matrix $\mathbf{G}$ of the hexaface is the upperright $2 \times 2$ block of matrix $\mathbf{M}_{\infty}$, and therefore the upper $2 \times 2$ block of the $6 \times 2$ matrix $\left(\mathbf{I}_{6}-\mathbf{A}\right)^{-1} \mathbf{B}$. Let us first calculate $\left(\mathbf{I}_{6}-\mathbf{A}\right)^{-1}$. It follows from decomposition (B.2) that

$$
\mathbf{I}_{6}-\mathbf{A}=\left(\begin{array}{ccc}
\mathbf{I} & -\mathbf{P} & \mathbf{0} \\
\mathbf{0} & \mathbf{I} & -\mathbf{C} \\
\mathbf{0} & -\mathbf{R} & \mathbf{I}
\end{array}\right)
$$

One can check by a straightforward direct multiplication that

$$
\left(\mathbf{I}_{6}-\mathbf{A}\right)^{-1}=\left(\begin{array}{ccc}
\mathbf{I} & \mathbf{P}(\mathbf{I}-\mathbf{C R})^{-1} & \mathbf{P C}(\mathbf{I}-\mathbf{R C})^{-1} \\
\mathbf{0} & (\mathbf{I}-\mathbf{C R})^{-1} & \mathbf{C}(\mathbf{I}-\mathbf{R C})^{-1} \\
\mathbf{0} & \mathbf{R}(\mathbf{I}-\mathbf{C R})^{-1} & (\mathbf{I}-\mathbf{R C})^{-1}
\end{array}\right) .
$$

Using the block decomposition of B, given in Eq. (B.3), we obtain

$$
\left(\mathbf{I}_{6}-\mathbf{A}\right)^{-1} \mathbf{B}=\left(\begin{array}{c}
\mathbf{S}+\mathbf{P C}(\mathbf{I}-\mathbf{R C})^{-1} \mathbf{X} \\
\mathbf{C}(\mathbf{I}-\mathbf{R C})^{-1} \mathbf{X} \\
(\mathbf{I}-\mathbf{R C})^{-1} \mathbf{X}
\end{array}\right) .
$$

$\left(\mathbf{I}_{6}-\mathbf{A}\right)^{-1} \mathbf{B}$ is a $6 \times 2$ matrix, whose upper $2 \times 2$ block is the global transfer matrix $\mathbf{G}$ :

$$
\mathbf{G}=\mathbf{S}+\mathbf{P C}(\mathbf{I}-\mathbf{R C})^{-1} \mathbf{X} .
$$

I-RC is expanded as

$$
\begin{aligned}
\mathbf{I}-\mathbf{R C} & =\left(\begin{array}{ll}
1 & 0 \\
0 & 1
\end{array}\right)-\left(\begin{array}{cc}
0 & r_{v} \\
r_{u} & 0
\end{array}\right) \cdot\left(\begin{array}{cc}
\tau & \rho \\
\rho^{\prime} & \tau^{\prime}
\end{array}\right) \\
& =\left(\begin{array}{cc}
1-r_{v} \rho^{\prime} & -r_{v} \tau^{\prime} \\
-r_{u} \tau & 1-r_{u} \rho
\end{array}\right) .
\end{aligned}
$$

Its determinant is

$$
D=\left(1-r_{u} \rho\right)\left(1-r_{v} \rho^{\prime}\right)-r_{u} r_{v} \tau \tau^{\prime},
$$

and its inverse is

$$
(\mathbf{I}-\mathbf{R C})^{-1}=\frac{1}{D}\left(\begin{array}{cc}
1-r_{u} \rho & r_{v} \tau^{\prime} \\
r_{u} \tau & 1-r_{v} \rho^{\prime}
\end{array}\right) .
$$

Thus, Eq. (B.5) becomes

$$
\begin{aligned}
\mathbf{G}= & \left(\begin{array}{cc}
0 & s_{u} \\
s_{v} & 0
\end{array}\right)+\frac{1}{D}\left(\begin{array}{cc}
p_{u} & 0 \\
0 & p_{v}
\end{array}\right) \cdot\left(\begin{array}{cc}
\tau & \rho \\
\rho^{\prime} & \tau^{\prime}
\end{array}\right) \\
& \cdot\left(\begin{array}{cc}
1-r_{u} \rho & r_{v} \tau^{\prime} \\
r_{u} \tau & 1-r_{v} \rho^{\prime}
\end{array}\right) \cdot\left(\begin{array}{cc}
x_{v} & 0 \\
0 & x_{u}
\end{array}\right),
\end{aligned}
$$

and we obtain the hexaface formula, with $D$ given by Eq. (B.6):

$\mathbf{G}=\left(\begin{array}{cc}p_{u} x_{v} \frac{\tau}{D} & s_{u}+p_{u} x_{u} \frac{\rho-r_{v}\left(\rho \rho^{\prime}-\tau \tau^{\prime}\right)}{D} \\ s_{v}+p_{v} x_{v} \frac{\rho^{\prime}-r_{u}\left(\rho \rho^{\prime}-\tau \tau^{\prime}\right)}{D} & p_{v} x_{u} \frac{\tau^{\prime}}{D}\end{array}\right)$.

\section{APPENDIX C: PARTICULAR TRANSFER MATRIX OF TRANSPARENT BIFACES}

In the present appendix, we propose detailed expressions for the particular transfer matrix of a transparent biface. Transparent bifaces may be flat interfaces, transparent layers, or superpositions of transparent layers and flat interfaces. Their particular transfer matrix depends on the position of the biface within the multiface, the geometry of illumination, and if the biface is at an external position, on the geometry of the capturing device.

Let us first consider a transparent biface being at a central position in a multiface and having the following fundamental transfer matrix:

$$
\left[\begin{array}{cc}
T(\theta) & R(\theta) \\
R^{\prime}(\theta) & T^{\prime}(\theta)
\end{array}\right],
$$

In order to have a regular multiface and be able to apply the compositional model, the transparent biface is necessarily bordered by Lambertian bifaces (see Section 4). Therefore, it receives Lambertian illuminations on its two faces. Its particular transfer matrix is obtained by integrating over the hemisphere each term of its fundamental transfer matrix [see Eq. (7)]:

$$
\begin{aligned}
L \downarrow & {\left[\begin{array}{cc}
T(\theta) & R(\theta) \\
R^{\prime}(\theta) & T^{\prime}(\theta)
\end{array}\right]_{\uparrow L} } \\
\quad & {\left[\begin{array}{cc}
\int_{\theta=0}^{\pi / 2} T(\theta) \sin 2 \theta \mathrm{d} \theta & \int_{\theta=0}^{\pi / 2} R(\theta) \sin 2 \theta \mathrm{d} \theta \\
\int_{\theta=0}^{\pi / 2} R^{\prime}(\theta) \sin 2 \theta \mathrm{d} \theta & \int_{\theta=0}^{\pi / 2} T^{\prime}(\theta) \sin 2 \theta \mathrm{d} \theta
\end{array}\right], }
\end{aligned}
$$

We now consider a transparent biface located at the upper position of a multiface. In order to ensure that the multiface is regular, it is necessarily bordered on its lower side by a Lambertian biface (see Section 4). Therefore, it 
Table 2. Particular Transfer Matrix of a Transparent Biface According to the Illumination and Measuring Geometries at Its Upper Side ${ }^{a}$

Directional light source at angle $\psi$ :

Capture:Integrating sphere

${ }^{\operatorname{dir}(\psi) \downarrow}\left[\begin{array}{ll}P(\theta) & S(\theta) \\ R(\theta) & X(\theta)\end{array}\right]_{\uparrow L}^{\uparrow s p h}=\left[\begin{array}{ll}P(\psi) & S(\psi) \\ \int_{R} & \int_{X}\end{array}\right]$

Lambertian light source:

Capture:Integrating sphere

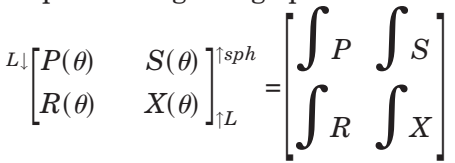

Radiance detector at angle $\psi^{\prime} \neq \psi$ :

$\operatorname{dir}(\psi) \downarrow\left[\begin{array}{ll}P(\theta) & S(\theta) \\ R(\theta) & X(\theta)\end{array}\right]_{\uparrow L}^{\uparrow r a d\left(\psi^{\prime}\right)}=\left[\begin{array}{lc}P(\psi) & 0 \\ \int R & \frac{X\left(\psi_{1}^{\prime}\right)}{\pi\left(n_{1} / n_{0}\right)^{2}}\end{array}\right]$

with $\psi_{1}^{\prime}=\arcsin \left(n_{0} \sin \psi^{\prime} / n_{1}\right)$

Radiance detector at angle $\psi^{\prime}$ :

$\operatorname{dir}(\psi) \downarrow\left[\begin{array}{ll}P(\theta) & S(\theta) \\ R(\theta) & X(\theta)\end{array}\right]_{\uparrow L}^{\uparrow \operatorname{rad}\left(\psi^{\prime}\right)}=\left[\begin{array}{ll}\int P & S\left(\psi^{\prime}\right) / \pi \\ \int R & \frac{X\left(\psi_{1}^{\prime}\right)}{\pi\left(n_{1} / n_{0}\right)^{2}}\end{array}\right]$

with $\psi_{1}^{\prime}=\arcsin \left(n_{0} \sin \psi^{\prime} / n_{1}\right)$

${ }^{a} n_{0}$ is the refractive index of the upper bordering medium (e.g., air) and $n_{1}$ of the lower bordering medium.

Table 3. Particular Transfer Matrix of a Transparent Biface According to the Illumination and Measuring Geometries at Its Lower Side ${ }^{a}$

Directional light source at angle $\psi$ :

Capture: Integrating sphere

${ }_{s p h \downarrow} \downarrow\left[\begin{array}{ll}X(\theta) & R(\theta) \\ S(\theta) & P(\theta)\end{array}\right]_{\uparrow \operatorname{dir}(\psi)}=\left[\begin{array}{ll}\int X & \int_{R} \\ S(\psi) & P(\psi)\end{array}\right]$

Lambertian light source:

Capture: Integrating sphere Integrating sphere

${ }_{s p h \downarrow}\left[\begin{array}{ll}X(\theta) & R(\theta) \\ S(\theta) & P(\theta)\end{array}\right]_{\uparrow L}=\left[\begin{array}{l}\int X \\ \int S \\ \int S\end{array}\right]$
Radiance detector at angle $\psi^{\prime} \neq \psi$ :

$$
\underset{\downarrow}{\operatorname{rad}\left(\psi^{\prime}\right) \downarrow}\left[\begin{array}{ll}
X(\theta) & R(\theta) \\
S(\theta) & P(\theta)
\end{array}\right]_{\uparrow \operatorname{dir}(\psi)}=\left[\begin{array}{cc}
\frac{X\left(\psi_{1}^{\prime}\right)}{\pi\left(n_{1} / n_{0}\right)^{2}} & \mathcal{J} R \\
0 & P(\psi)
\end{array}\right]
$$

with $\psi_{1}^{\prime}=\arcsin \left(n_{0} \sin \psi^{\prime} / n_{1}\right)$

Radiance detector at angle $\psi^{\prime}$ :

$\operatorname{rad}_{\downarrow}\left[\begin{array}{cc}X(\theta) & R(\theta) \\ S(\theta) & P(\theta)\end{array}\right]_{\uparrow L}=\left[\begin{array}{ll}\frac{X\left(\psi_{1}^{\prime}\right)}{\pi\left(n_{1} / n_{0}\right)^{2}} & \int_{R} \\ S\left(\psi^{\prime}\right) / \pi & \int_{P}\end{array}\right]$

with $\psi_{1}^{\prime}=\arcsin \left(n_{0} \sin \psi^{\prime} / n_{1}\right)$

${ }^{a} n_{0}$ is the refractive index of the lower bordering medium (e.g., air) and $n_{1}$ of the upper bordering medium.

receives a Lambertian illumination on its lower face. Its fundamental transfer matrix is defined as

$$
\left[\begin{array}{ll}
P(\theta) & S(\theta) \\
R(\theta) & X(\theta)
\end{array}\right] .
$$

Its particular transfer matrices are presented in Table 2 for a directional or a Lambertian light source, and for an integrating sphere or a radiance detector as a capturing device located on its upper side (for the derivation of the expressions, see $[9,10])$. On the fundamental transfer matrix, the upper and lower illumination geometries are specified respectively by a top-left superscript with a symbol $(\downarrow)$ and by a bottom-right subscript with symbol $(\uparrow)$. Symbols $L$ and $\operatorname{dir}(\psi)$ stand, respectively, for Lambertian and for directional with incident angle $\psi$. The capturing device is specified by a top-right subscript with symbol $(\uparrow)$. Symbols $\operatorname{rad}(\psi)$ and $s p h$ stand, respectively, for radiance detector with observation angle $\psi$ and for integrating sphere. Notation $\int g$ stands for $\int_{\theta=0}^{\pi / 2} g(\theta) \sin 2 \theta \mathrm{d} \theta$. Terms $n_{0}$ and $n_{1}$ are the refractive indices of respectively the upper and lower side media bordering the upper transparent biface (e.g. the interface air-medium). Angle $\psi_{1}^{\prime}$ is related to observation angle $\psi^{\prime}$ by Snell's laws:

$$
n_{1} \sin \psi_{1}^{\prime}=n_{0} \sin \psi^{\prime} \text {. }
$$

Table 3 is similar to Table 2 when the transparent biface is at the lowest position within a multiface.

Note that for matte prints, when a $45^{\circ} / 0^{\circ}$ geometry is used, a rough print-air interface can be considered as being flat since its transmittance for incident light is at $45^{\circ}$, its transmittance for exiting light is at $0^{\circ}$, and its internal reflectance is very close to that of a flat interface [21].

\section{REFERENCES}

1. P. Kubelka and F. Munk, "Ein Beitrag zur Optik der Farbanstriche," Z. Tech. Phys. (Leipzig) 12, 593-601 (1931).

2. P. Kubelka, "New contributions to the optics of intensely light-scattering material. Part I," J. Opt. Soc. Am. 38, 448-457 (1948).

3. W. E. Vargas and G. A. Niklasson, "Applicability conditions of the Kubelka-Munk theory," Appl. Opt. 36, 5580-5586 (1997).

4. P. Kubelka, "New contributions to the optics of intensely 
light-scattering materials. Part II: Nonhomogeneous layers," J. Opt. Soc. Am. 44, 330-335 (1954).

5. M. Born and E. Wolf, Principle of Optics, 7th ed. (Pergamon, 1999).

6. J. L. Saunderson, "Calculation of the color pigmented plastics," J. Opt. Soc. Am. 32, 727-736 (1942).

7. J. W. Ryde, "The scattering of light by turbid media," Proc. R. Soc. London A131, 451-464 (1931).

8. G. Kortüm, "Phenomenological theories of absorption and scattering of tightly packed particles," in Reflectance Spectroscopy (Springer Verlag, 1969), pp. 103-168.

9. M. Hébert and R. D. Hersch, "A reflectance and transmittance model for recto-verso halftone prints," J. Opt. Soc. Am. A 22, 1952-1967 (2006).

10. M. Hébert, "Compositional model for predicting multilayer reflectances and transmittances in color reproduction," Ph.D. Thesis No. 3576 (Ecole Polytechnique Fédérale de Lausanne, 2006).

11. F. C. Williams and F. R. Clapper, "Multiple internal reflections in photographic color prints," J. Opt. Soc. Am. 29, 595-599 (1953).

12. J. D. Shore and J. P. Spoonhower, "Reflection density in photographic color prints: generalizations of the Williams-Clapper transform," J. Imaging Sci. Technol. 45, 484-488 (2001).
13. L. Simonot, M. Hébert, and R. D. Hersch, "Extension of the Williams-Clapper model to stacked nondiffusing colored coatings with different refractive indices," J. Opt. Soc. Am. A 23, 1432-1441 (2006).

14. F. R. Clapper and J. A. C. Yule, "The effect of multiple internal reflections on the densities of halftone prints on paper," J. Opt. Soc. Am. 43, 600 (1953).

15. W. R. McCluney, Introduction to Radiometry and Photometry (Artech House, 1994).

16. D. B. Judd, "Fresnel reflection of diffusely incident light," J. Res. Natl. Bur. Stand. 29, 329-332 (1942).

17. H.-H. Perkampus, Encyclopedia of Spectroscopy (VCH, 1995).

18. W. J. Stewart, Introduction to the Numerical Solution of Markov Chains (Princeton Univ. Press, 1994).

19. M. Hébert and R. D. Hersch, "Classical print reflection models: a radiometric approach," J. Imaging Sci. Technol. 48, 363-374 (2004).

20. C. D. Meyer, Matrix Analysis and Applied Linear Algebra (SIAM, 2000), p. 701.

21. M. Hébert and R. D. Hersch, "Extending the Clapper-Yule model to rough printing supports," J. Opt. Soc. Am. A 22, 1952-1967 (2005). 